\title{
Identification of a Putative DNA-Binding Protein in Arabidopsis That Acts as a Susceptibility Hub and Interacts With Multiple Pseudomonas syringae Effectors
}

\author{
Karl J. Schreiber, ${ }^{1}$ and Jennifer D. Lewis ${ }^{1,2,+}$ \\ ${ }^{1}$ Department of Plant and Microbial Biology, University of California Berkeley, Berkeley, CA 94720-3102, U.S.A. \\ ${ }^{2}$ Plant Gene Expression Center, United States Department of Agriculture, Albany, CA 94710-1105, U.S.A. \\ Accepted 24 December 2020.
}

\begin{abstract}
Phytopathogens use secreted effector proteins to suppress host immunity and promote pathogen virulence, and there is increasing evidence that the host-pathogen interactome comprises a complex network. To identify novel interactors of the Pseudomonas syringae effector HopZ1a, we performed a yeast two-hybrid screen that identified a previously uncharacterized Arabidopsis protein that we designate HopZ1a interactor 1 (ZIN1). Additional analyses in yeast and in planta revealed that ZIN1 also interacts with several other $P$. syringae effectors. We show that an Arabidopsis loss-of-function zin1 mutant is less susceptible to infection by certain strains of $P$. syringae, while overexpression of ZIN1 results in enhanced susceptibility. Functionally, ZIN1 exhibits topoisomerase-like activity in vitro. Transcriptional profiling of wild-type and zin1 Arabidopsis plants inoculated with $P$. syringae indicated that while ZIN1 regulates a wide range of pathogen-responsive biological processes, the list of genes more highly expressed in zin1 versus wild-type plants is particularly enriched for ribosomal protein genes. Altogether, these data illuminate ZIN1 as a potential susceptibility hub that interacts with multiple effectors to influence the outcome of plant-microbe interactions.
\end{abstract}

Keywords: Arabidopsis, Pseudomonas syringae, susceptibility factor, transcriptional regulation, type III effector

The molecular landscape of plant-microbe interactions is both dynamic and complex. The initial entry of potential pathogens into plant tissues can be detected extracellularly by pattern recognition receptors that recognize conserved pathogen-

Sequence data for the genes examined in this study are available in the National Center for Biotechnology Information database under accession numbers NM_125375 (ZIN1) and NM_114527 (GRAS1).

${ }^{\dagger}$ Corresponding author: J. D. Lewis; jdlewis@berkeley.edu

Funding: This research was funded by United States Department of Agriculture Agricultural Research Service grants 2030-21000-046-00D and 2030-21000-050-00D, National Science Foundation Directorate for Biological Sciences grant IOS-1557661, and National Institutes of Health shared instrumentation grant 1S10OD010786-01.

*The $\boldsymbol{e}$-Xtra logo stands for "electronic extra" and indicates supplementary figures and supplementary tables are published online.

The author(s) declare no conflict of interest.

(1) $\circledast \Theta$ Copyright $\odot 2021$ The Author(s). This is an open access article distributed under the CC BY-NC-ND 4.0 International license. associated molecular patterns (PAMPs) and stimulate PAMPtriggered immunity (PTI) to prevent the establishment of an infection (Peng et al. 2018; Saijo et al. 2018). As a countermeasure, many pathogens introduce effector proteins into the intracellular space of host cells in order to suppress PTI and allow pathogens to proliferate (Khan et al. 2018; Lewis et al. 2009; Varden et al. 2017). This has selected for the evolution of various intracellular nucleotide-binding, leucine-rich repeat (NLR) proteins in plants capable of recognizing the activity of effectors and eliciting a more intense immune response, known as effector-triggered immunity (ETI) (Lolle et al. 2020; Schreiber et al. 2016). The evolution of additional effectors that suppress ETI will restore pathogen virulence. As such, diverse and ever-changing collections of pathogen effectors and host immune receptors determine the outcome of plant-microbe interactions.

The suppression of host immunity by effector proteins is accomplished in a variety of subcellular locations, including the nucleus. Within this organelle, there are several different potential mechanisms by which host gene expression could be broadly altered to promote pathogen virulence. Numerous effectors target and inactivate host transcription factors by facilitating their degradation, altering their subcellular localization, introducing posttranslational modifications, or physically associating with a target to impair the assembly or activity of a transcriptional protein complex (Kim et al. 2013; McLellan et al. 2013; Qin et al. 2018; Yang et al. 2017). A different approach is exemplified by the family of transcription activator-like (TAL) effectors, which directly bind to the promoters of host genes such as sugar transporters in order to promote pathogen growth (Verdier et al. 2012). The host transcriptome can also be manipulated by effectors that alter histone acetylation (Kong et al. 2017; Li et al. 2018). Indeed, epigenetic regulation and chromatin remodeling are important mediators of plant immunity (Ding and Wang 2015).

As host plants evolve in the presence of pathogens, effector targets may be lost or modified. If an effector normally inactivates a target, the loss of that target could mimic effector activity and contribute to increased susceptibility to infection. For targets that act as negative regulators of immunity or are activated by effectors to promote virulence, loss or mutation of the target will eliminate an avenue of exploitation by pathogens, thus reducing disease susceptibility. These targets are referred to as susceptibility factors (van Schie and Takken 2014) and have garnered interest as potentially significant mediators of durable disease resistance. Mildew resistance locus o (Mlo) genes encode some of the most well-studied susceptibility factors. Barley loss-of-function mlo mutants exhibit 
non-race specific resistance to infection by the powdery mildew pathogen Blumeria graminis f. sp. hordei, and the mutation of Mlo orthologs in other plant species also confers powdery mildew resistance (Kusch and Panstruga 2017). Importantly, MLO2 from Arabidopsis interacts with the Pseudomonas syringae effector HopZ2 and is necessary for the promotion of virulence by HopZ2 (Lewis et al. 2012). Transcription factors can also be susceptibility factors. For example, the basic helixloop-helix protein CHL1 from potato is indirectly upregulated by the Phytophthora infestans effector AVR2 to promote pathogen proliferation, and a knockdown of its expression results in reduced susceptibility to infection (Turnbull et al. 2017, 2019). Additional transcriptional regulators have been identified in high-throughput interaction screens as direct targets of effectors that can be considered susceptibility factors (Weßling et al. 2014).

Phytopathogens typically harbor multiple effectors, many of which remain to be fully characterized in terms of their molecular targets in host cells as well as the biological consequences of these interactions. The effector-host protein interactome has previously been surveyed through selective pairwise screening of specific effectors against a defined set of Arabidopsis proteins (Mukhtar et al. 2011; Weßling et al. 2014). In this study, we sought to identify Arabidopsis proteins that interact with the $P$. syringae effector protein HopZ1a, which is an acetyltransferase whose enzymatic activity contributes both to its virulence activity and its recognition by the NLR ZAR1 (Lee et al. 2012; Lewis et al. 2010, 2013). Since HopZ1a was not included in previous interactome screens, we lack a complete list of host proteins targeted by this effector. Using large-scale yeast two-hybrid screens, we identified and recently described an APETALA2-domain transcription factor that interacts with HopZ1a (Schreiber et al. 2020). Here, we focused on another interactor, which is a previously uncharacterized protein that we have designated as HopZ1a interactor 1 (ZIN1). We verified that HopZ1a and ZIN1 interact in planta. Interestingly, Arabidopsis zin1 T-DNA knockout plants are less susceptible to infection by specific strains of $P$. syringae compared with wild-type (WT) plants. Conversely, ZIN1 overexpression is associated with increased susceptibility to $P$. syringae. We showed that ZIN1 interacts with multiple $P$. syringae effectors. We also found that ZIN1 exhibits topoisomerase-like activity in vitro. Global transcriptional profiling demonstrated that ZIN1 influences a wide range of biological processes that are manipulated by $P$. syringae in an effector-dependent manner, with a particularly significant impact on the expression of genes associated with the machinery of protein translation. Altogether, these data indicate that ZIN1 is a susceptibility factor in Arabidopsis that may be targeted by multiple effector proteins as a mechanism for promoting pathogen virulence.

\section{RESULTS}

Identification and preliminary characterization of ZIN1.

To define the HopZ1a interactome, a yeast two-hybrid interaction screen was previously conducted using a Quantitative Interactor Screen Sequencing (QIS-Seq) approach, which involves large-scale screens coupled with next-generation sequencing (Lewis et al. 2012). Through this process, we identified the Arabidopsis protein ZIN1 (encoded by At5g59830) as a putative HopZ1a interactor. We confirmed this association in planta by bimolecular fluorescence complementation (BiFC) and found that both WT HopZ1a and an acetyltransferase-deficient mutant (HopZ1a ${ }^{\mathrm{C} 216 \mathrm{~A}}$ ) could associate with ZIN1 (Fig. 1). Within the HopZ family of effectors, HopZ3 could also interact with ZIN1, while HopZ2 could not.
We used a translational fusion of ZIN1 and full-length yellow fluorescent protein (YFP) to verify that ZIN1 is nuclearlocalized and that coexpression with various HopZ effectors does not alter this subcellular localization pattern (Supplementary Fig. S1).

To determine whether ZIN1 has a role in plant immunity, we obtained an Arabidopsis T-DNA knockout line bearing a transposon insertion in the first exon of ZIN1. Importantly, zin1 plants are phenotypically identical to WT plants in terms of

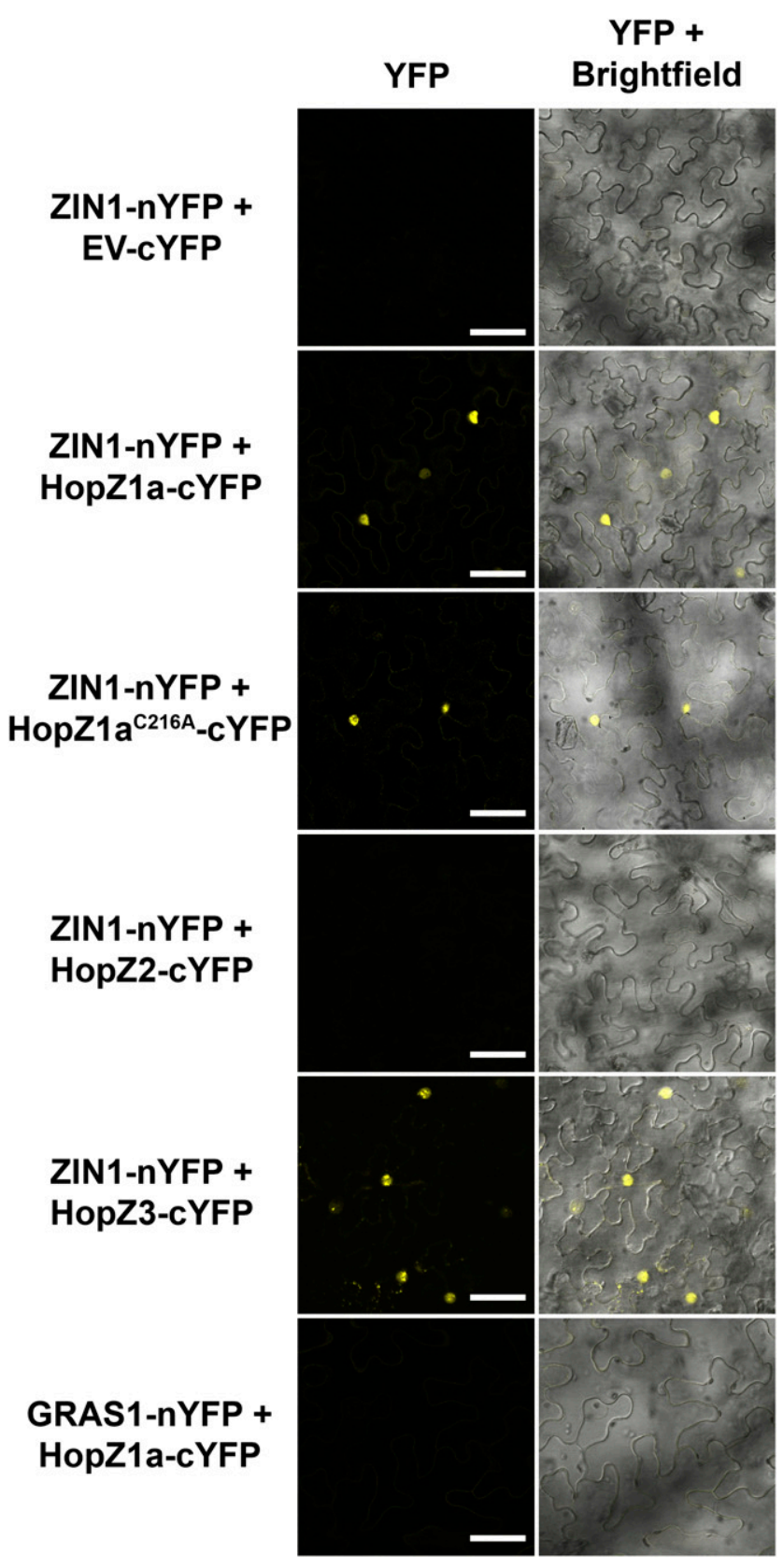

Fig. 1. Evaluation of interactions between ZIN1 and members of the HopZ effector family through bimolecular fluorescence complementation assays. The assays included wild-type HopZ1a, a catalytic mutant of HopZ1a (C216A), HopZ2, and HopZ3. An empty expression vector (EV) construct and a GRAS family transcription factor (GRAS1, At3g46600) were included as negative controls. Proteins were transiently expressed in Nicotiana benthamiana leaves by agroinfiltration as fusions to an $\mathrm{N}$ - or C-terminal portion of yellow fluorescent protein (nYFP or cYFP, respectively). Expression was induced by $20 \mu \mathrm{M}$ dexamethasone at $24 \mathrm{~h}$ postinoculation and images were collected $24 \mathrm{~h}$ later. Scale bars $=50 \mu \mathrm{m}$. 
growth and overall morphology (Supplementary Fig. S2). We infiltrated these plants with either $P$. syringae pv. tomato DC3000 (DC3000), P. cannabina pv. alisalensis ES4326 (ES4326, formerly $P$. syringae pv. maculicola ES4326, or $P$. syringae pv. cilantro 0788-9 (0788-9). The highly virulent strain DC3000 multiplied to $\log 6.1$ in WT plants, and its growth was reduced by 0.8 log colony-forming units (CFU) per square centimeter in zinl plants, representing a reduction of $86 \%$ (Fig. 2A). The weakly virulent strain 0788-9 reached a population of $\log 4.5$ on WT hosts but generally grew $0.4 \log$
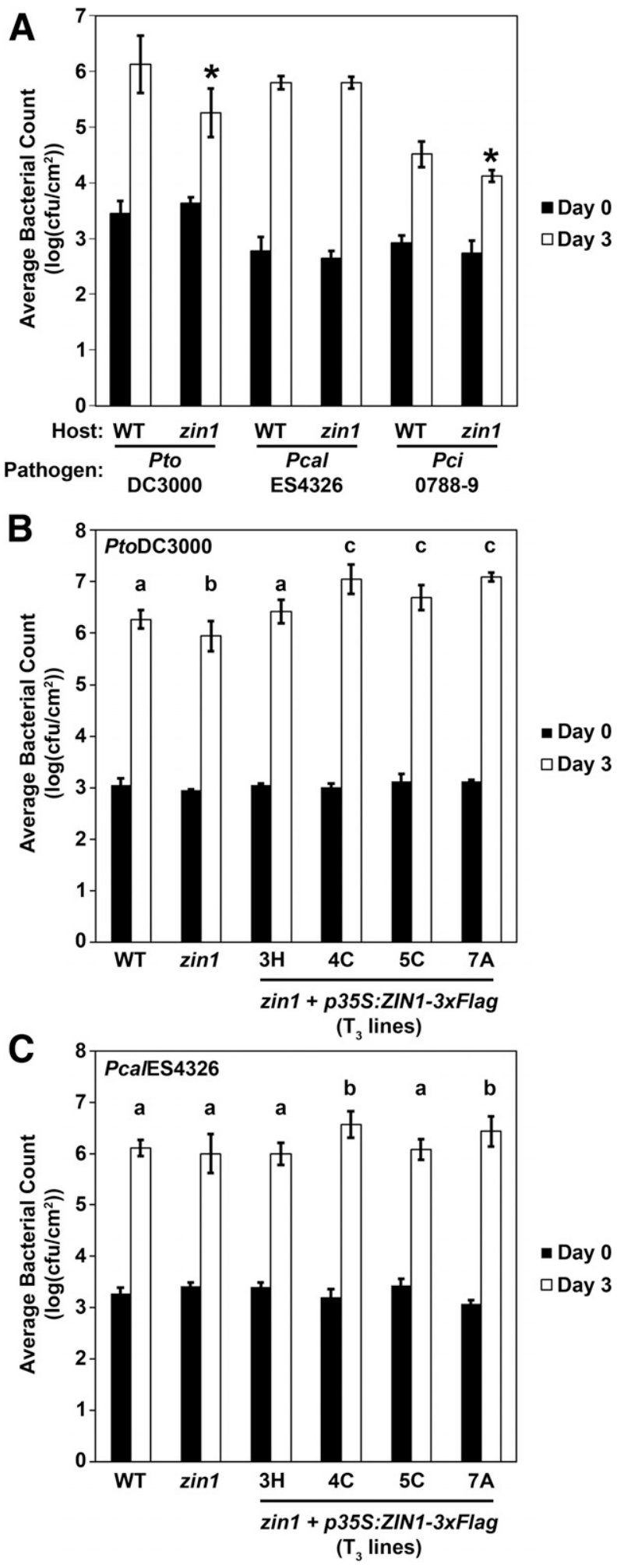

CFU per square centimeter less in zinl plants, which amounts to a $60 \%$ reduction in bacterial growth. Interestingly, the growth of ES4326 did not differ significantly between WT and zin1 plants. In addition, these two host genotypes supported equivalent populations of DC3000 expressing HopZ1a, indicating that ZAR1-mediated ETI is not altered in zin1 plants (Supplementary Fig. S3). We also generated transgenic zin1 plants in which ZIN1-3xFlag was constitutively expressed from a cauliflower mosaic virus $35 \mathrm{~S}$ promoter. We tested plants from the $\mathrm{T}_{3}$ generation and found that they were generally even more susceptible to infection with DC3000 than WT plants (Fig. 2B; Supplementary Fig. S4). Some transgenic lines also exhibited enhanced susceptibility to ES4326 (Fig. 2C). Altogether, these data suggest that ZIN1 does influence plant immunity with regards to interactions with $P$. syringae.

ZIN1 interacts with multiple effectors.

While ZIN1 was identified through its interaction with HopZ1a, it should be noted that DC3000, ES4326, and 0788-9 all lack HopZ1a. We therefore hypothesized that additional effectors may interact with ZIN1 at the host-pathogen interface. To test this idea, we performed yeast two-hybrid assays using pairwise mating to evaluate the interactions between ZIN1 and 29 effectors from DC3000. We observed relatively strong interactions with the effectors HopAF1 and HopE1 in yeast, while HopA1, HopB1, HopC1, HopN1, and HopQ1-1 interacted weakly with ZIN1 (Fig. 3A). A GRAS family transcription factor (encoded by At3g46600) was included as a negative control to confirm that the effectors were not prone to nonspecific interactions (Supplementary Fig. S5). We confirmed the ZIN1-effector associations in planta by BiFC (Fig. 3B), although the ZIN1-HopQ1-1 association was only observed with ZIN1 fused to the C-terminal portion of YFP and HopQ1-1 fused to the YFP N-terminus. As with the HopZ family effectors, the nuclear localization of ZIN1 was generally preserved in the presence of the interacting DC3000 effectors, with the exception of HopAF1.

\section{ZIN1 sequence analysis.}

Little information is available in any sequence databases regarding the biological function of ZIN1, so we sought to derive functional predictions from analyses of the ZIN1 sequence. Within this protein, the sole conserved sequence element comprises a tify (TIF[F/Y]XG) domain-binding domain (TDBD) (Fig. 4A) (Bai et al. 2011; Kazan and Manners 2012; Pauwels et al. 2010). Structural homology modeling with Phyre 2 identified two short regions near the C-terminus of ZIN1 that resemble a SAND domain (Bottomley et al. 2001) and a topoisomerase enzyme (Patel et al. 2006) (Fig. 4A;

Fig. 2. Arabidopsis HopZ1a interactor 1 (ZIN1) influences plant immunity. A, Growth of Pseudomonas spp. in wild type (WT) versus zinl knockout plants. The leaves of six-week-old plants were inoculated by pressure infiltration with $1 \times 10^{5}$ - $\mathrm{CFU} / \mathrm{ml}$ suspensions of $P$. syringae pv. tomato DC3000 (Pto DC3000), P. cannabina pv. alisalensis ES4326 (Pcal ES4326), or $P$. syringae pv. cilantro 0788-9 (Pci 0788-9), and bacterial populations were quantified at 3 days postinoculation. Error bars represent the standard deviation from the mean of eight samples. For each strain, an asterisk indicates a statistically significant difference between WT and zin 1 plants as determined by a Student's $t$ test $(\alpha=0.05)$. B and $\mathbf{C}$, Influence of ZIN1 overexpression on susceptibility to PtoDC3000 or PcalES4326, respectively. The $\mathrm{T}_{3}$ generation of four independent lines of transgenic Arabidopsis zin1 plants constitutively expressing ZIN1 (zin1 + p35S:ZIN1$3 x$ Flag) was compared with wild-type and zin 1 plants. Inoculations were performed as described above. Error bars represent the standard deviation from the mean of ten samples. Letters above datapoints indicate statistical significance groups as determined by pairwise Student's $t$ tests $(\alpha=0.05)$. 
Supplementary Fig. S6), modeled with 25 and $73 \%$ confidence, respectively. Alignments of the corresponding sequences indicate that the topoisomerase-like region of ZIN1 includes some but not all of the key catalytic residues found in the most similar topoisomerase structure from the bacterium Deinococcus radiodurans (Patel et al. 2006) (Supplementary Fig. S6). The ZIN1 SAND-like domain lacks the conserved TPXXFE and KDWK motifs that characterize functional SAND domain-containing proteins (Bottomley et al. 2001; Carles et al. 2005), but this region includes the TDBD sequence and thus could be functionally relevant. Indeed, the C-terminal sequence of ZIN1 is highly conserved throughout the plant kingdom (Supplementary Fig. S7). It is also interesting to note that, of the 18 TDBD-containing proteins in Arabidopsis, 16 contain additional conserved domains, most commonly a PHD finger (Sanchez and Zhou 2011) (Supplementary Fig. S8). The two "TDBD only" proteins include ZIN1 and a predicted N-lysine methyltransferase (At5g13660). Using structural homology modeling, we found that the central region of the putative methyltransferase bore significant similarity to bacterial adhesin proteins (e.g., Protein Data Bank ID 3S6L) and that the C-terminus could be modeled on topoisomerase enzymes, albeit at even lower confidence than observed with ZIN1. Overall, therefore, ZIN1 appears to be a unique and conserved plant protein.

Beyond the C-terminus, the majority of the ZIN1 protein is predicted to be disordered (Fig. 4A). Within the disordered regions, computational analyses predict short stretches of potential secondary structure as well as several possible proteinprotein interaction interfaces. The Eukaryotic Linear Motif resource (Gouw et al. 2018) identified many highly conserved short linear interaction motifs (SLiMs) (Supplementary Table S1), while the ANCHOR2 (Mészáros et al. 2018), MoRFpred (Disfani et al. 2012), and MoRF chibi (Malhis et al. 2016) algorithms predicted four longer interfaces known as molecular recognition factors (MoRFs) (Fig. 4A). To determine which region or regions of ZIN1 may be targeted by effectors, we tested HopZ1a against various ZIN1 truncations in yeast twohybrid assays. We compared full-length ZIN1 to constructs lacking the topoisomerase-like domain, one lacking both the topoisomerase-like domain and the TDBD/SAND-like domain, and one comprising just these two C-terminal domains. These analyses indicated that HopZ1a likely binds ZIN1 in a region that includes the TDBD/SAND-like domain and some of the disordered sequence N-terminal to this domain (Fig. 4B; Supplementary Fig. S9). In addition, HopZ1a reproducibly interacted with the $\mathrm{ZIN1}_{1-353}$ truncation more strongly than with full-length ZIN1, suggesting that the topoisomerase-like domain may inhibit the association with HopZ1a to some degree.

\section{ZIN1 exhibits topoisomerase-like activity.}

The prediction of a topoisomerase domain in ZIN1 by structural homology modeling led us to evaluate this putative

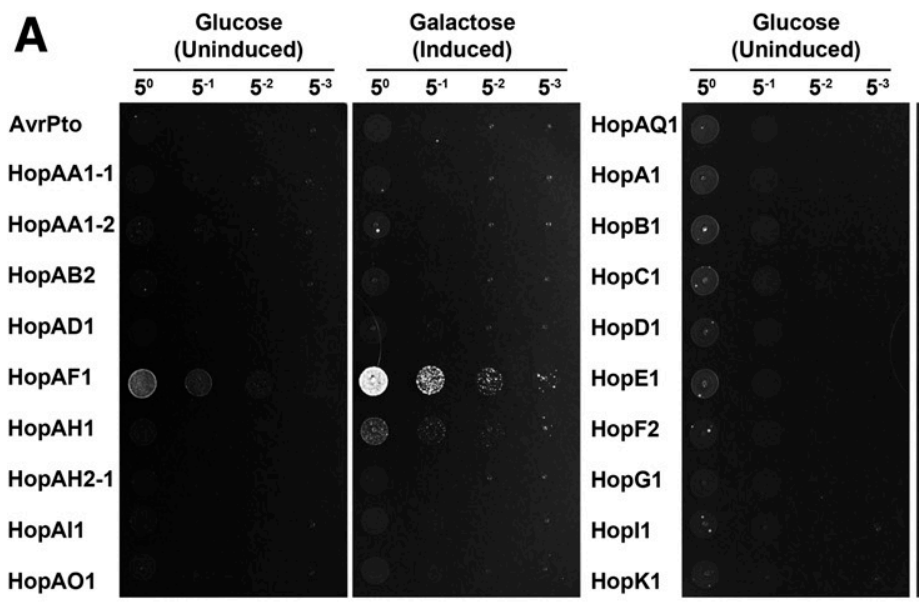

B BiFC: ZIN1-nYFP + Effector-cYFP
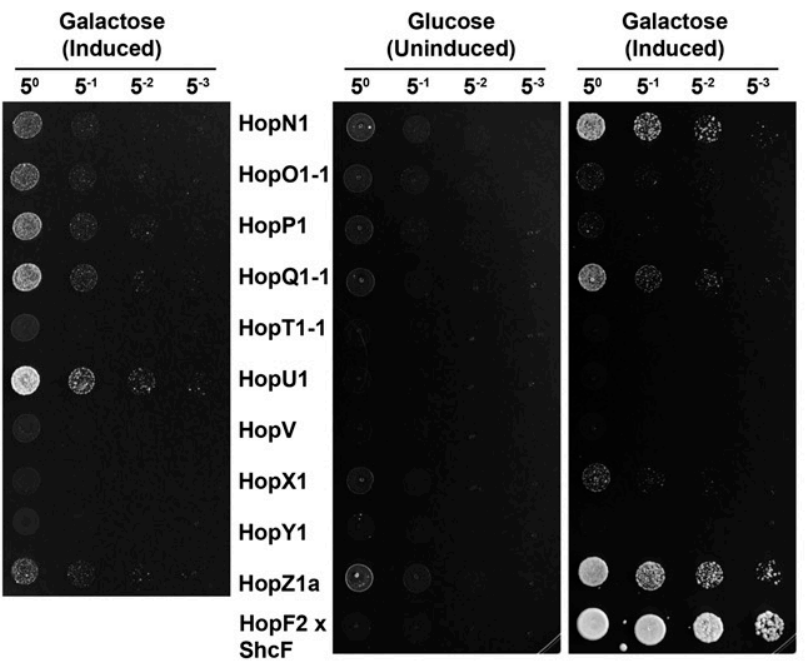

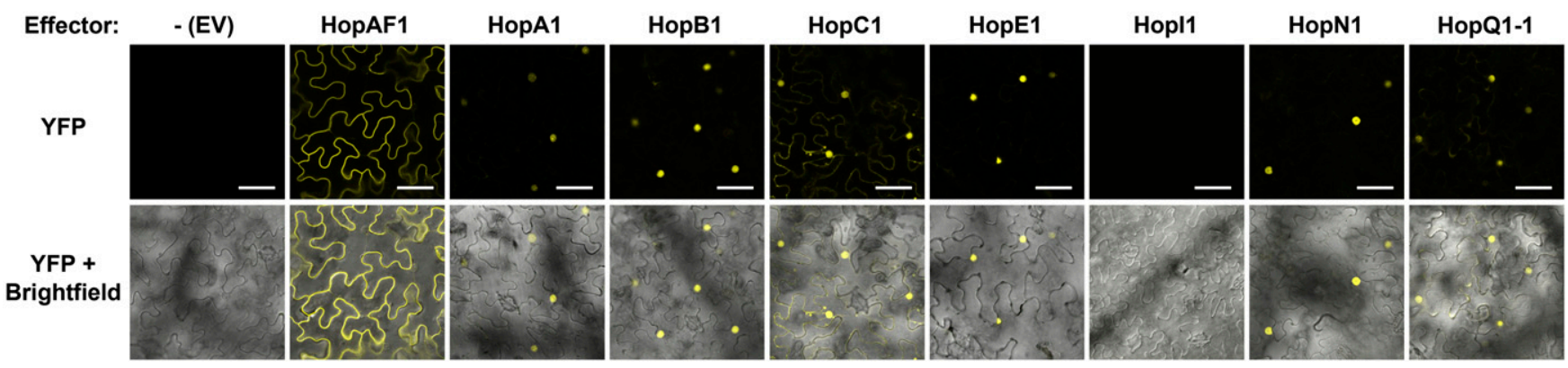

Fig. 3. Arabidopsis HopZ1a interactor 1 (ZIN1) interacts with multiple Pseudomonas syringae effector proteins. A, Yeast two-hybrid analyses of ZIN1-effector interactions. Effectors were constitutively expressed in yeast and ZIN1 was expressed from a galactose-inducible promoter. In addition to HopZ1a, 29 effectors from P. syringae pv. tomato DC3000 (DC3000) were tested against ZIN1 by pairwise yeast mating. The DC3000 genes HopF2 and ShcF were included as a positive control, based on their previously documented strong interaction (Shan et al. 2004). Yeast suspensions at optical density at $600 \mathrm{~nm}=2 \mathrm{and}$ three fivefold serial dilutions were spotted on nutritional selection media, and images were generally captured after 3 days of growth. B, Evaluation of ZIN1-effector interactions by bimolecular fluorescence complementation (BiFC) assays in Nicotiana benthamiana. An empty expression vector (EV) construct and HopI 1 were included as negative controls. Proteins were transiently expressed in N. benthamiana leaves by agroinfiltration as fusions to an N-or C-terminal portion of yellow fluorescent protein (nYFP or cYFP, respectively). Expression was induced by $20 \mu \mathrm{M}$ dexamethasone at $24 \mathrm{~h}$ postinoculation, and images were collected $24 \mathrm{~h}$ later. Scale bars $=50 \mu \mathrm{m}$. All experiments were performed at least three times with similar results. The ZIN1-HopQ1-1 interaction was only observed with ZIN1-cYFP and HopQ1-1-nYFP, while all other protein associations were observed in both directions of split-YFP tags. 
enzymatic activity in vitro. We purified full-length ZIN1 $\left(\mathrm{ZIN} 1_{1-425}\right)$ as well as a truncation lacking both the TDBD/SAND-like and topoisomerase-like regions (ZIN1 $\left.1_{1-305}\right)$, then incubated these proteins with a purified plasmid. If ZIN1 has topoisomerase activity, it should facilitate the unwinding of DNA and, thus, alter the plasmid conformations observed following gel electrophoresis, specifically reducing the abundance of the supercoiled conformation in favor of more relaxed conformations (Manohar et al. 2017). Indeed, full-length ZIN1 facilitated a significant shift from supercoiled to linear and nicked/relaxed plasmid conformations (Fig. 5). This shift was less pronounced following incubation with the $\mathrm{ZIN1}_{1-305}$ truncation, suggesting that the deleted region does contribute significantly to the plasmid relaxation phenotype. An equivalent amount of purified $\beta$-glucuronidase (GUS) was included as a negative control and did not alter the conformations of the test plasmid. Finally, we noted that this assay could be performed in reaction buffer lacking ATP, which is compatible with the conclusion that ZIN1 resembles enzymes from the type I topoisomerase family.

\section{Global transcriptomic analysis of the contribution}

of ZIN1 to plant immunity.

Presuming that ZIN1 is a DNA-binding protein with apparent topoisomerase-like activity, the targeting of ZIN1 by effectors could mediate broad impacts on gene expression in plants. To probe this hypothesis, we inoculated leaves of WT and zinl plants with $1 \times 10^{8} \mathrm{CFU} / \mathrm{ml}$ suspensions of either WT DC3000, a DC3000 mutant that is unable to secrete effectors (DC3000 $\Delta h r c C$ ), or $10 \mathrm{mM} \mathrm{MgCl}_{2}$ as a control. Tissues were collected at 6 and $12 \mathrm{~h}$ postinoculation (hpi) for gene expression profiling by Tag-Seq, which is a 3 '-end RNA sequencing approach (Lohman et al. 2016; Meyer et al. 2011). To minimize artifacts in data analysis, we aligned sequencing reads to the Arabidopsis genome using either STAR or HISAT2 and

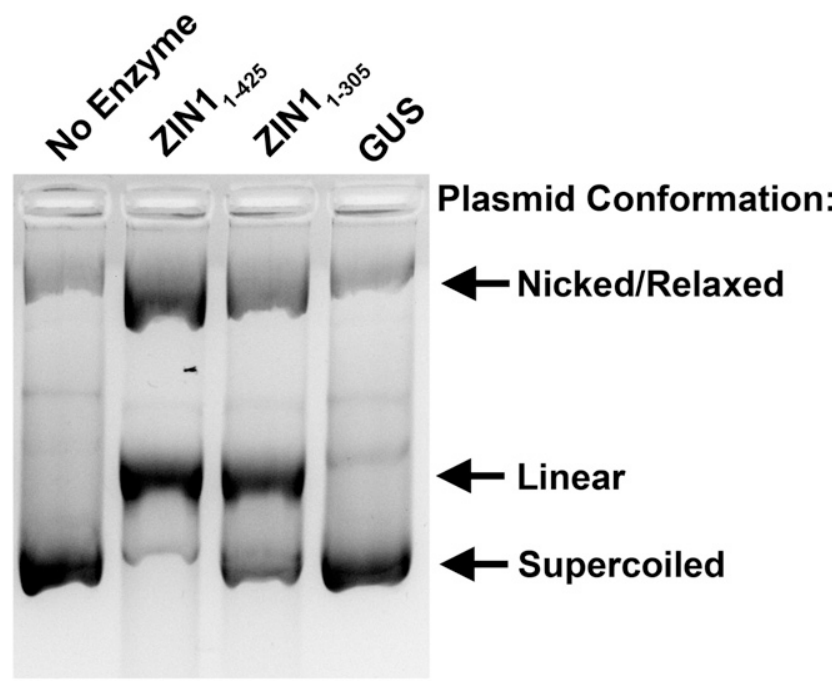

Fig. 5. Arabidopsis HopZ1a interactor 1 (ZIN1) exhibits topoisomerase-like activity in vitro. A 4-nM solution of purified plasmid was incubated at $30^{\circ} \mathrm{C}$ for $1 \mathrm{~h}$ with reaction buffer (no enzyme) or approximately $1 \mu \mathrm{g}$ of purified full-length ZIN1 (ZIN1 1-425), a C-terminal ZIN1 truncation (ZIN11-305), or $\beta$-glucuronidase (GUS) as an additional negative control. Similar electrophoresis images were captured from three different experiments.

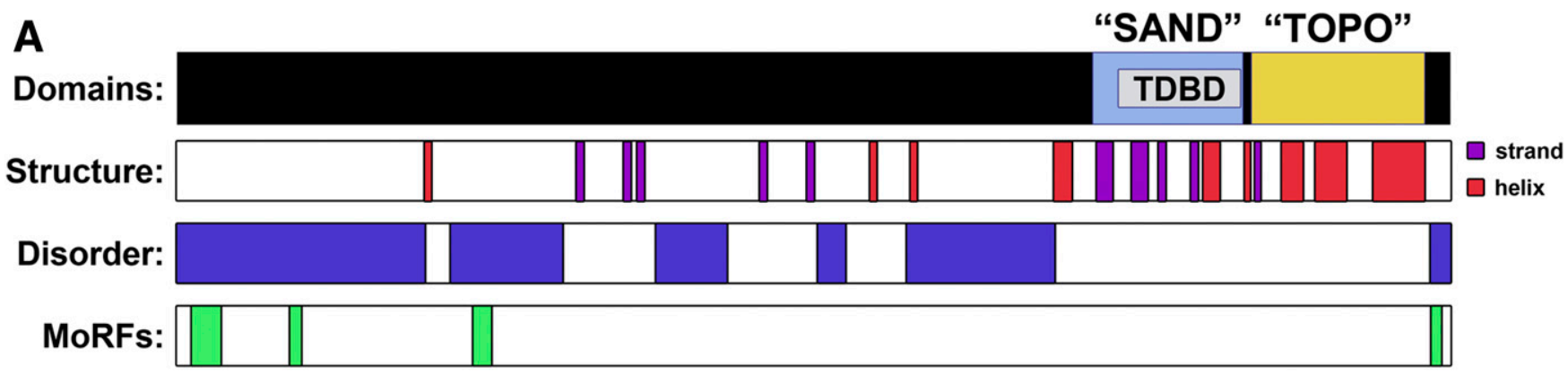

B

HopZ1a x:

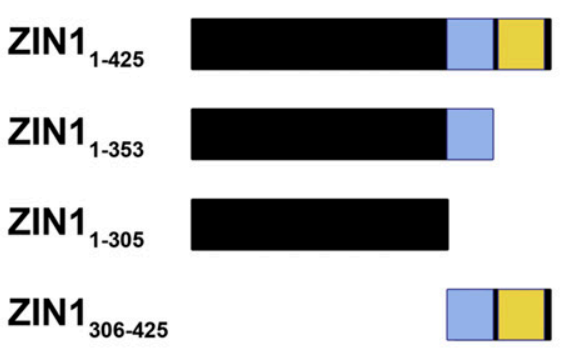

Glucose (Uninduced)

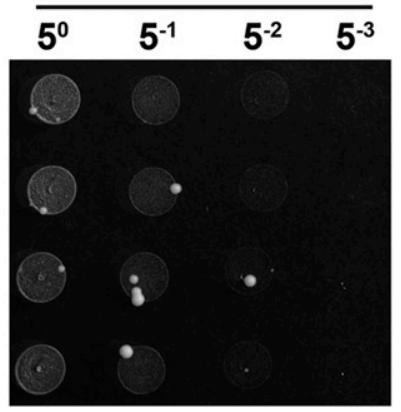

Galactose (Induced)

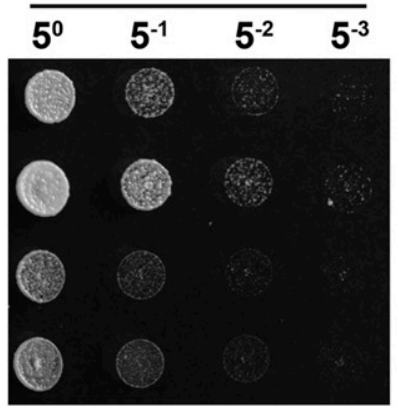

Fig. 4. Predicted sequence features of Arabidopsis HopZ1a interactor 1 (ZIN1) and domain interactions with the effector HopZ1a. A, Computational predictions of functionally relevant regions within ZIN1. A tify domain-binding domain (TDBD) was identified by querying the Pfam database, while structural homology modeling with Phyre 2 revealed regions that resemble a SAND domain and a topoisomerase (TOPO) domain. Secondary protein structure was predicted using PSIPRED, as illustrated with purple and red boxes representing $\beta$-strands and $\alpha$-helices, respectively. A consensus of results from PONDRFIT-VSL2 and DISOPRED3 defined regions of predicted structural disorder (blue boxes). Within these regions, putative interaction interfaces known as molecular recognition features (MoRFs) were predicted using ANCHOR2, MoRFpred, and MoRF chibi (green boxes). B, Yeast two-hybrid analyses of the interaction between HopZ1a and specific regions of ZIN1. HopZ1a was constitutively expressed in yeast and ZIN1 was expressed from a galactose-inducible promoter. Yeast suspensions at optical density at $600 \mathrm{~nm}=2$ and three fivefold serial dilutions were spotted on nutritional selection media, and images were generally captured after 3 days of growth. The experiment was performed at least three times with similar results. 
identified differentially expressed genes with either the edgeR or limma-voom algorithms. We generated four datasets using the two aligners and two differential expression algorithms and assembled final lists of differentially expressed genes as the consensus among these four datasets. When we compared gene expression in inoculated tissues to control tissues infiltrated with $10 \mathrm{mM} \mathrm{MgCl}$ at $6 \mathrm{hpi,}$ 2,972 genes exhibited statistically significant changes in expression under one or more conditions, including 1,322 upregulated and 1,660 downregulated genes (Fig. 6), with an overlap of 10 genes that were either up- or downregulated depending on the experimental condition. At $12 \mathrm{hpi}$, this same comparison revealed 4,655 differentially expressed genes (2,249 upregulated and 2,458 downregulated, with 52 conditionally overlapping genes). Within each combination of host genotype and pathogen inoculation, $\log _{2}$-fold changes in gene expression at the two timepoints were moderately correlated ( $r=0.64$ to 0.69 ) (Supplementary Fig. S10).

We identified a small number of genes as being differentially expressed between WT and zinl plants in the absence of infection
(Supplementary Table S2). These included two genes with lower expression in zinl plants relative to WT at 6 hpi. At $12 \mathrm{hpi}, 25$ genes were more highly expressed in zinl plants, while 19 were expressed at lower levels. The expression of one gene (At1g64040) was consistently at least eightfold lower in zinl plants relative to WT at both 6 and 12 hpi (Supplementary Table S2).

With regards to responses to pathogen infection, we examined specific subsets of differentially expressed genes. Based on our observations of ZIN1 interacting with multiple effectors, we focused on genes with ZIN1- and effector-dependent expression patterns as well as genes uniquely differentially expressed in a zinl background in an effector-dependent manner. Within these groups, there was a statistically insignificant overlap between the lists of differentially expressed genes at 6 and $12 \mathrm{hpi}$, although the $\log _{2}$-fold change values exhibited moderate correlations $(r=0.66$ to 0.67$)$ between the two timepoints (Supplementary Fig. S11). To functionally describe these genes, gene ontology (GO) term enrichment was assessed using the online Database for Annotation,
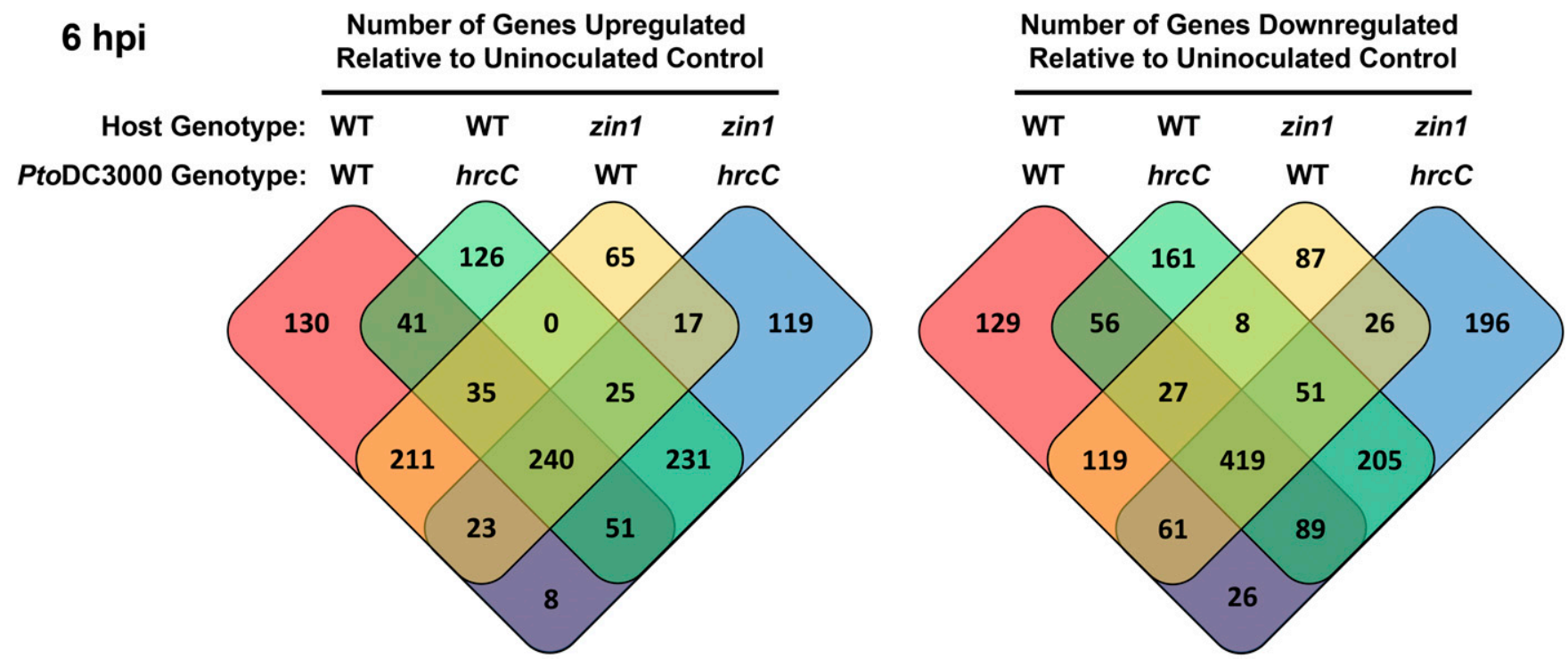

\section{$12 \mathrm{hpi}$ \\ Number of Genes Upregulated Relative to Uninoculated Control}

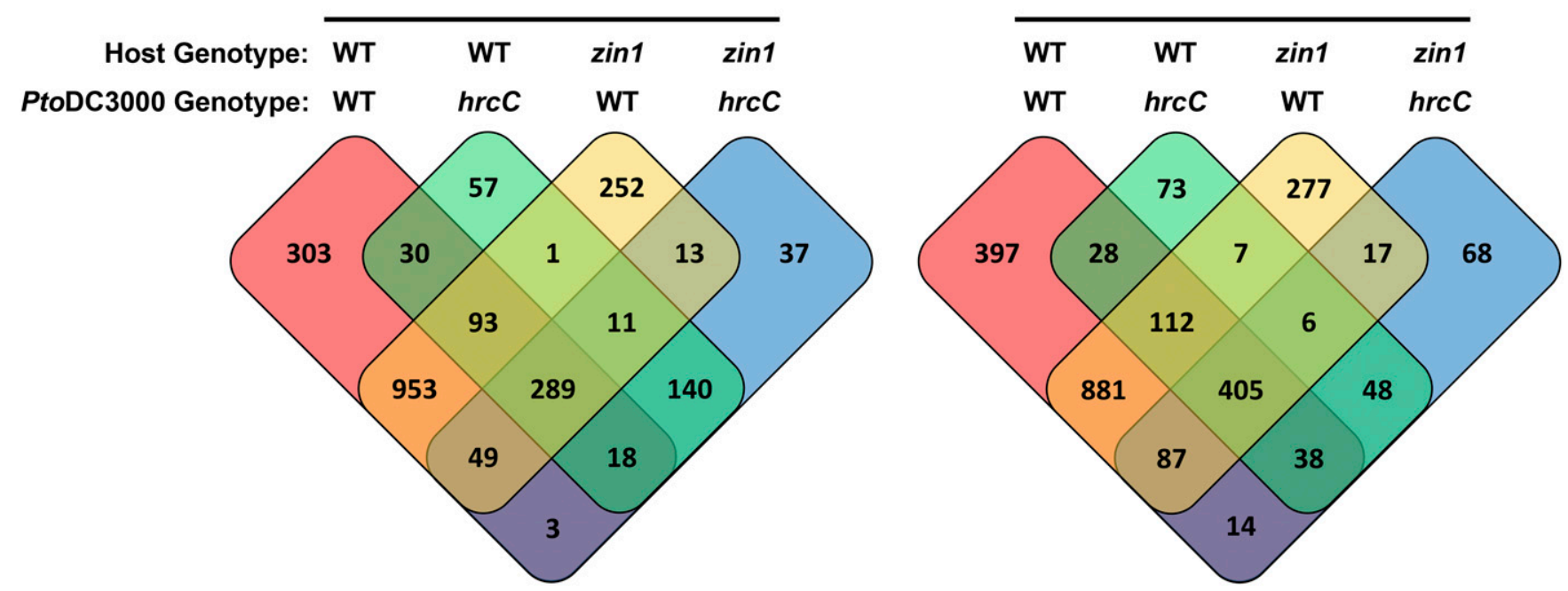

\section{Number of Genes Downregulated}

Relative to Uninoculated Control

Fig. 6. Summary of RNA-seq analysis of the influence of HopZ1a interactor 1 (ZIN1) on transcriptional responses to pathogen infection in Arabidopsis. Either wild-type (WT) or zin1 mutant Arabidopsis plants were infiltrated with $10 \mathrm{mM} \mathrm{MgCl} 2$ or $1 \times 10^{8}$-CFU/ml suspensions of either WT Pseudomonas syringae pv. tomato DC3000 or a type III secretion-deficient DC3000 mutant (hrcC). Tissues were collected at 6 and 12 h postinoculation (hpi) for analysis by RNAsequencing. Differentially expressed genes were identified by comparing gene expression in pathogen-inoculated tissues versus $\mathrm{MgCl}_{2}$-infiltrated control tissues. Venn diagrams indicate overlaps in the lists of differentially expressed genes between the four host-pathogen genotype combinations tested. 
Table 1. Gene ontology (GO) enrichment analysis of effector-dependent APETRIZ (altered pathogen-elicited transcriptional response in zin1) genes at $12 \mathrm{~h}$ postinoculation

\begin{tabular}{|c|c|c|c|c|}
\hline Category & Term & Count & Percent of list & $P$ value \\
\hline \multicolumn{5}{|c|}{ Calculated APETRIZ difference $>0$} \\
\hline Biological process & GO:0006412: translation & 64 & 9.222 & $4.20 \mathrm{E}-14$ \\
\hline Biological process & GO:0019253: reductive pentose-phosphate cycle & 9 & 1.297 & $6.60 \mathrm{E}-06$ \\
\hline Biological process & GO:0015979: photosynthesis & 16 & 2.305 & 4.82E-04 \\
\hline Biological process & GO:0042254: ribosome biogenesis & 18 & 2.594 & $5.65 \mathrm{E}-04$ \\
\hline Biological process & GO:0010114: response to red light & 9 & 1.297 & 8.77E-04 \\
\hline Biological process & GO:0010218: response to far red light & 8 & 1.153 & 0.002 \\
\hline Biological process & GO:0009735: response to cytokinin & 19 & 2.738 & 0.003 \\
\hline Biological process & GO:0009773: photosynthetic electron transport in photosystem I & 5 & 0.720 & 0.003 \\
\hline Biological process & GO:0015977: carbon fixation & 4 & 0.576 & 0.004 \\
\hline Biological process & GO:0055114: oxidation-reduction process & 57 & 8.213 & 0.004 \\
\hline Biological process & GO:0034220: ion transmembrane transport & 5 & 0.720 & 0.010 \\
\hline Biological process & GO:0032544: plastid translation & 4 & 0.576 & 0.014 \\
\hline Biological process & GO:0009637: response to blue light & 7 & 1.009 & 0.019 \\
\hline Biological process & GO:0015994: chlorophyll metabolic process & 3 & 0.432 & 0.020 \\
\hline Biological process & GO:0009992: cellular water homeostasis & 5 & 0.720 & 0.020 \\
\hline Biological process & GO:0006810: transport & 19 & 2.738 & 0.026 \\
\hline Biological process & $\begin{array}{l}\text { GO:0080158: chloroplast ribulose bisphosphate carboxylase complex } \\
\text { biogenesis }\end{array}$ & 3 & 0.432 & 0.032 \\
\hline Biological process & GO:0042742: defense response to bacterium & 21 & 3.026 & 0.033 \\
\hline Biological process & GO:0006414: translational elongation & 8 & 1.153 & 0.038 \\
\hline Biological process & GO:0009658: chloroplast organization & 10 & 1.441 & 0.043 \\
\hline Biological process & GO:0009056: catabolic process & 4 & 0.576 & 0.047 \\
\hline Molecular function & GO:0003735: structural constituent of ribosome & 65 & 9.366 & $3.37 \mathrm{E}-15$ \\
\hline Molecular function & GO:0019843: rRNA binding & 20 & 2.882 & $8.15 \mathrm{E}-08$ \\
\hline Molecular function & GO:0016984: ribulose-bisphosphate carboxylase activity & 4 & 0.576 & 8.14E-04 \\
\hline Molecular function & GO:0003729: mRNA binding & 33 & 4.755 & 0.002 \\
\hline Molecular function & GO:0015250: water channel activity & 6 & 0.865 & 0.003 \\
\hline Molecular function & GO:0005507: copper ion binding & 17 & 2.450 & 0.010 \\
\hline Molecular function & GO:0003723: RNA binding & 44 & 6.340 & 0.011 \\
\hline Molecular function & GO:0015254: glycerol channel activity & 5 & 0.720 & 0.016 \\
\hline Molecular function & GO:0016491: oxidoreductase activity & 21 & 3.026 & 0.016 \\
\hline Molecular function & $\begin{array}{l}\text { GO:0016620: oxidoreductase activity, acting on the aldehyde or oxo group of } \\
\text { donors, NAD or NADP as acceptor }\end{array}$ & 4 & 0.576 & 0.019 \\
\hline Molecular function & GO:0051287: NAD binding & 8 & 1.153 & 0.028 \\
\hline Cellular compartment & GO:0009507: chloroplast & 240 & 34.582 & $4.90 \mathrm{E}-19$ \\
\hline Cellular compartment & GO:0009535: chloroplast thylakoid membrane & 59 & 8.501 & $1.27 \mathrm{E}-16$ \\
\hline Cellular compartment & GO:0009579: thylakoid & 46 & 6.628 & $4.42 \mathrm{E}-16$ \\
\hline Cellular compartment & GO:0009570: chloroplast stroma & 80 & 11.527 & $6.08 \mathrm{E}-15$ \\
\hline Cellular compartment & GO:0005840: ribosome & 53 & 7.637 & $9.69 \mathrm{E}-13$ \\
\hline Cellular compartment & GO:0009941: chloroplast envelope & 69 & 9.942 & $2.11 \mathrm{E}-12$ \\
\hline Cellular compartment & GO:0009534: chloroplast thylakoid & 33 & 4.755 & $5.61 \mathrm{E}-09$ \\
\hline Cellular compartment & GO:0022627: cytosolic small ribosomal subunit & 23 & 3.314 & $1.74 \mathrm{E}-08$ \\
\hline Cellular compartment & GO:0022626: cytosolic ribosome & 34 & 4.899 & $2.23 \mathrm{E}-08$ \\
\hline Cellular compartment & GO:0048046: apoplast & 32 & 4.611 & $2.07 \mathrm{E}-04$ \\
\hline Cellular compartment & GO:0009543: chloroplast thylakoid lumen & 13 & 1.873 & $2.12 \mathrm{E}-04$ \\
\hline Cellular compartment & GO:0016020: membrane & 89 & 12.824 & $2.50 \mathrm{E}-04$ \\
\hline Cellular compartment & GO:0005773: vacuole & 49 & 7.061 & $2.96 \mathrm{E}-04$ \\
\hline Cellular compartment & GO:0030095: chloroplast photosystem II & 6 & 0.865 & $4.12 \mathrm{E}-04$ \\
\hline Cellular compartment & GO:0022625: cytosolic large ribosomal subunit & 20 & 2.882 & $4.33 \mathrm{E}-04$ \\
\hline Cellular compartment & GO:0009573: chloroplast ribulose bisphosphate carboxylase complex & 4 & 0.576 & 8.04E-04 \\
\hline Cellular compartment & GO:0010598: NAD $(\mathrm{P}) \mathrm{H}$ dehydrogenase complex (plastoquinone) & 5 & 0.720 & 0.002 \\
\hline Cellular compartment & GO:0009523: photosystem II & 7 & 1.009 & 0.003 \\
\hline Cellular compartment & GO:0031977: thylakoid lumen & 9 & 1.297 & 0.003 \\
\hline Cellular compartment & GO:0005730: nucleolus & 32 & 4.611 & 0.009 \\
\hline Cellular compartment & GO:0031428: box C/D snoRNP complex & 4 & 0.576 & 0.009 \\
\hline Cellular compartment & GO:0005618: cell wall & 27 & 3.890 & 0.032 \\
\hline Cellular compartment & GO:0015934: large ribosomal subunit & 8 & 1.153 & 0.033 \\
\hline Cellular compartment & GO:0005623: cell & 14 & 2.017 & 0.041 \\
\hline Cellular compartment & GO:0010319: stromule & 6 & 0.865 & 0.044 \\
\hline Cellular compartment & GO:0015030: Cajal body & 4 & 0.576 & 0.047 \\
\hline \multicolumn{5}{|c|}{ Calculated APETRIZ difference $<0$} \\
\hline Biological process & GO:0006468: protein phosphorylation & 34 & 6.427 & $2.83 \mathrm{E}-04$ \\
\hline Biological process & GO:0009626: plant-type hypersensitive response & 10 & 1.890 & 0.002 \\
\hline Biological process & $\begin{array}{l}\text { GO:0009862: systemic acquired resistance, salicylic acid mediated signaling } \\
\text { pathway }\end{array}$ & 5 & 0.945 & 0.002 \\
\hline Biological process & GO:0009615: response to virus & 7 & 1.323 & 0.003 \\
\hline Biological process & GO:0010200: response to chitin & 12 & 2.268 & 0.003 \\
\hline Biological process & GO:0048194: Golgi vesicle budding & 4 & & 0.003 \\
\hline
\end{tabular}


Table 1. (Continued from previous page)

\begin{tabular}{|c|c|c|c|c|}
\hline Category & Term & Count & Percent of list & $P$ value \\
\hline Biological process & GO:0018105: peptidyl-serine phosphorylation & 8 & 1.512 & 0.004 \\
\hline Biological process & GO:0006886: intracellular protein transport & 16 & 3.025 & 0.004 \\
\hline Biological process & GO:0035556: intracellular signal transduction & 11 & 2.079 & 0.007 \\
\hline Biological process & GO:0046777: protein autophosphorylation & 11 & 2.079 & 0.007 \\
\hline Biological process & GO:0050832: defense response to fungus & 15 & 2.836 & 0.008 \\
\hline Biological process & GO:0009617: response to bacterium & 10 & 1.890 & 0.011 \\
\hline Biological process & GO:0007165: signal transduction & 18 & 3.403 & 0.013 \\
\hline Biological process & GO:0009751: response to salicylic acid & 11 & 2.079 & 0.023 \\
\hline Biological process & GO:0006511: ubiquitin-dependent protein catabolic process & 15 & 2.836 & 0.023 \\
\hline Biological process & GO:0000304: response to singlet oxygen & 3 & 0.567 & 0.030 \\
\hline Biological process & GO:0010541: acropetal auxin transport & 3 & 0.567 & 0.030 \\
\hline Biological process & GO:0009738: abscisic acid-activated signaling pathway & 12 & 2.268 & 0.030 \\
\hline Biological process & GO:0006816: calcium ion transport & 4 & 0.756 & 0.032 \\
\hline Biological process & GO:0030433: ER-associated ubiquitin-dependent protein catabolic process & 5 & 0.945 & 0.037 \\
\hline Biological process & GO:0031047: gene silencing by RNA & 4 & 0.756 & 0.044 \\
\hline Biological process & GO:0010197: polar nucleus fusion & 4 & 0.756 & 0.044 \\
\hline Biological process & GO:0006457: protein folding & 17 & 3.214 & 0.045 \\
\hline Biological process & GO:0016192: vesicle-mediated transport & 12 & 2.268 & 0.047 \\
\hline Molecular function & GO:0005516: calmodulin binding & 23 & 4.348 & $1.13 \mathrm{E}-05$ \\
\hline Molecular function & GO:0005524: ATP binding & 91 & 17.202 & $2.59 \mathrm{E}-05$ \\
\hline Molecular function & GO:0009931: calcium-dependent protein serine/threonine kinase activity & 7 & 1.323 & $2.26 \mathrm{E}-04$ \\
\hline Molecular function & GO:0005509: calcium ion binding & 21 & 3.970 & $3.79 \mathrm{E}-04$ \\
\hline Molecular function & GO:0004683: calmodulin-dependent protein kinase activity & 7 & 1.323 & $5.47 \mathrm{E}-04$ \\
\hline Molecular function & GO:0016301: kinase activity & 38 & 7.183 & 0.003 \\
\hline Molecular function & GO:0004012: phospholipid-translocating ATPase activity & 4 & 0.756 & 0.008 \\
\hline Molecular function & $\begin{array}{l}\text { GO:0042626: ATPase activity, coupled to transmembrane movement of } \\
\text { substances }\end{array}$ & 7 & 1.323 & 0.013 \\
\hline Molecular function & GO:0004674: protein serine/threonine kinase activity & 28 & 5.293 & 0.017 \\
\hline Molecular function & GO:0017137: Rab GTPase binding & 5 & 0.945 & 0.018 \\
\hline Molecular function & GO:0030276: clathrin binding & 5 & 0.945 & 0.018 \\
\hline Molecular function & $\begin{array}{l}\text { GO:0015662: ATPase activity, coupled to transmembrane movement of ions, } \\
\text { phosphorylative mechanism }\end{array}$ & 4 & 0.756 & 0.019 \\
\hline Molecular function & GO:0016887: ATPase activity & 12 & 2.268 & 0.027 \\
\hline Molecular function & GO:0046527: glucosyltransferase activity & 3 & 0.567 & 0.032 \\
\hline Molecular function & GO:0005515: protein binding & 76 & 14.367 & 0.036 \\
\hline Molecular function & GO:0004672: protein kinase activity & 19 & 3.592 & 0.037 \\
\hline Molecular function & GO:0005198: structural molecule activity & 7 & 1.323 & 0.039 \\
\hline Molecular function & GO:0051539: 4 iron, 4 sulfur cluster binding & 6 & 1.134 & 0.046 \\
\hline Cellular compartment & GO:0005886: plasma membrane & 132 & 24.953 & $1.42 \mathrm{E}-05$ \\
\hline Cellular compartment & GO:0005634: nucleus & 250 & 47.259 & $1.57 \mathrm{E}-05$ \\
\hline Cellular compartment & GO:0000502: proteasome complex & 11 & 2.079 & $3.90 \mathrm{E}-04$ \\
\hline Cellular compartment & GO:0005737: cytoplasm & 140 & 26.465 & 0.003 \\
\hline Cellular compartment & GO:0030126: COPI vesicle coat & 4 & 0.756 & 0.009 \\
\hline Cellular compartment & GO:0000139: Golgi membrane & 19 & 3.592 & 0.017 \\
\hline Cellular compartment & GO:0005622: intracellular & 26 & 4.915 & 0.020 \\
\hline Cellular compartment & GO:0005839: proteasome core complex & 5 & 0.945 & 0.020 \\
\hline Cellular compartment & GO:0005788: endoplasmic reticulum lumen & 5 & 0.945 & 0.043 \\
\hline Cellular compartment & GO:0012505: endomembrane system & 6 & 1.134 & 0.043 \\
\hline Cellular compartment & GO:0080008: Cul4-RING E3 ubiquitin ligase complex & 8 & 1.512 & 0.046 \\
\hline Cellular compartment & GO:0008540: proteasome regulatory particle, base subcomplex & 4 & 0.756 & 0.048 \\
\hline
\end{tabular}

Visualization and Integrated Discovery (Huang et al. 2009a and b). At both 6 and $12 \mathrm{hpi}$, we detected moderate enrichment (at $P<0.05$ ) of a broad range of metabolic functions (Supplementary Tables S3 to S6). The most notable patterns of enrichment were found at $12 \mathrm{hpi}$ in the cellular compartment category, where ZIN1-, effector-dependent upregulated gene products were most likely to be nuclear-localized, while the downregulated gene list was enriched for chloroplast proteins. Genes that are downregulated in a zinl-, effector-dependent manner appear to be most often distributed among the Golgi apparatus, chloroplast, or plasma membrane.

Another noteworthy observation from the GO term analysis was that zinl plants do not seem to mount a stronger immune response than WT plants upon exposure to DC3000. A small number of defense-related genes are enriched at $6 \mathrm{hpi}$ in the ZIN1-, effector-dependent group, although they are found in both the up- and downregulated gene lists (Supplementary
Tables S3 and S4). Immune responses are not represented by any of the enriched GO terms at 12 hpi. Moreover, we examined specific marker genes of different aspects of plant immunity, including PTI, systemic acquired resistance (SAR), and phytohormone signaling, yet found few differences in gene expression patterns between WT and zinl plants at either timepoint (Supplementary Tables S7 and S8). As such, the reduced growth of $P$. syringae in zinl plants is more likely due to the loss of an important virulence target rather than the induction of more intense defense responses.

Analyses of ZIN1-dependent transcriptional responses.

Up to this point, we had identified differentially expressed genes within host genotypes by comparing expression in treated plants to control plants, but we also sought to perform quantitative analyses between genotypes to more directly compare the responses of WT and zin1 plants to $P$. syringae. We modeled 
these comparisons after previous studies of SAR, which occurs when a localized pathogen infection stimulates the release of one or more signaling molecules to distal tissues, resulting in reduced susceptibility to infection throughout the plant (Klessig et al. 2018; Shine et al. 2019). For these studies, the genes of greatest interest are those whose expression is not significantly altered in distal tissues after local infection but which are primed for more dramatic transcriptional changes following inoculation of these distal tissues. Generally, SAR experiments comprise different combinations of local and distal leaf inoculations. Within this context, Bernsdorff et al. (2016) defined genes as primed in distal leaves if the difference between normalized read counts of local $_{\text {inoculated }} /$ distal $_{\text {inoculated }}$ and local $_{\text {inoculated }} /$ distal $_{\text {mock }}$ treatments is statistically greater than the difference between local $l_{\text {mock }} /$ distal $_{\text {inoculated }}$ and local $l_{\text {mock }} /$ distal $_{\text {mock }}$ treatments. For our experiment, the analogous calculation would be (zin $\left.1_{\text {inoculated }}-z i n 1_{\text {mock }}\right)$ versus $\left(\mathrm{WT}_{\text {inoculated }}\right.$ $\left.-\mathrm{WT}_{\text {mock }}\right)$ and would identify genes that we refer to as APETRIZ (altered pathogen-elicited transcriptional response in zinl) genes. This analysis enabled us to statistically compare gene expression values between host genotypes while correcting for any variations due to genetic background or priming effect. A calculated difference of greater than zero would result if a gene is either more strongly upregulated in zinl or less strongly downregulated in zinl versus WT and vice versa for differences of less than zero. Using a Mann-Whitney two-sided $U$ test $(\alpha=0.005)$, we identified 558 APETRIZ genes at $6 \mathrm{~h}$ after WT $P$. syringae inoculation in zinl relative to WT plants (Supplementary Table S9). For DC3000 $4 h r c$-inoculated plants, 770 genes were considered statistically different by these same criteria (Supplementary Table S10), including 98 genes that were also identified in the WT DC3000 analysis. We filtered the list of APETRIZ genes from DC3000-inoculated plants by removing genes that were also identified as APETRIZ in DC3000 $h r c C$-inoculated plants, thus generating a list of effector-dependent APETRIZ genes. This list included 231 genes with calculated differences greater than zero (">0") and 229 genes with differences less than zero ("<0") (Supplementary Table S11). At 12 hpi, there were 700 " >0" and 529 " $<0$ " effector-dependent APETRIZ genes (Supplementary Tables S12 to S14).

We examined the effector-dependent APETRIZ gene lists and found that a small number of GO terms were moderately enriched at 6 hpi (Supplementary Table S15), while the results were much more striking at 12 hpi. For the " $>0$ " gene set, the biological process term "translation" was strongly enriched $(p \approx$ $10^{-14}$ ) at $12 \mathrm{hpi}$, paralleled by significant enrichment of the terms "structural constituent of the ribosome" and "rRNA binding" in the molecular function category (Table 1). Many of the proteins encoded by " $>0$ " genes are localized to the chloroplast as well as the cytosolic ribosome (Table 1 ). The " $<0$ " gene set exhibited lower enrichment overall, but the terms "protein phosphorylation," "calmodulin binding," "ATP binding," "plasma membrane," and "nucleus" were highly represented (Table 1).

These analyses highlighted a number of potentially interesting genes, and we sought to validate the expression of a subset of these genes by reverse transcription quantitative PCR (RT-qPCR). We replicated our initial experiment by infiltrating WT and zin1 mutant Arabidopsis plants with WT DC3000, DC3000 $\Delta h r c C$, or $10 \mathrm{mM}$ $\mathrm{MgCl}_{2}$ as a control. In addition, to investigate how HopZ1a might specifically affect gene expression, we expressed this effector in an otherwise effectorless DC3000 polymutant (DC3000 D36E) (Wei et al. 2015). We infiltrated these bacteria into either zarl or zarlzinl mutant plants to avoid the activation of cell death responses resulting from HopZ1a recognition. We ultimately focused on three genes that exhibited reproducibly altered ZIN1-dependent gene expression patterns (Fig. 7). These genes encode a protein phosphatase (At1g64040), a ribosomal protein S25 family protein (At4g34555), and a ribosomal protein L7Ae/L30e/S12e/Gadd45 family protein (At2g32060) (APETRIZ statistics can be found in Supplementary Table S12). Relative to the respective $\mathrm{MgCl}_{2}-$ treated controls, the expression of At1g64040 was lower in zin1 versus WT plants following inoculation with either WT DC3000 or DC3000 $\Delta h r c C$ (Fig. 7). These differences were not statistically significant in zarl or zarlzinl plants infiltrated with DC3000 D36E with or without hopZla. This suggests that the full complement of DC3000 effectors, which does not include HopZ1a, can influence ZIN1-dependent host gene expression in a different manner than HopZ1a alone. We also confirmed our observation from the RNAsequencing data that At1g64040 is expressed at a much lower level (approximately 12-fold) in zinl plants relative to WT in the absence of pathogen infiltration (Supplementary Fig. S12). When comparing uninoculated zarlzinl to zarl plants, the reduction in At1g64040 expression was less dramatic (1.3-fold) but still statistically significant. For the ribosomal protein genes At4g34555 and At2g32060, exposure to various genotypes of DC3000 led to reduced gene expression in WT/zarl plants relative to $\mathrm{MgCl}_{2}$ treatment, while these genes were less strongly suppressed or even induced in zinl/zarlzinl mutants (Fig. 7). In most cases, these statistically significant differences were effector-dependent.

\section{DISCUSSION}

The outcome of plant-pathogen interactions is strongly influenced by the activities of multiple pathogen effector proteins on a variety of targets in host cells. Some of this complexity is highlighted by our identification and characterization of ZIN1. In particular, we found that a zinl T-DNA line was less susceptible to $P$. syringae pv. tomato $\mathrm{DC} 3000$ and $P$. syringae pv. cilantro 0788-9, while the growth of ES4326 was similar in WT and zin1 plants. The effector repertoires of these three strains overlap substantially (Supplementary Fig. S13), including the ZIN1interacting effector HopQ1-1. At the same time, many effectors are unique to each strain, and it is possible that one or more effectors in ES4326 could compensate for the potential growth deficit incurred by the absence of ZIN1. An alternative explanation is that PTI is enhanced in zinl plants and that this response is suppressed by one or more of the effectors unique to ES4326. This hypothesis seems less likely, however, given that the expression of several PTI marker genes did not differ significantly between WT and zin 1 plants following exposure to $P$. syringae. Instead, we propose that ZIN1 is a virulence target and that its absence in a zinl plant eliminates one mechanism of effectormediated virulence promotion. As a supporting corollary, we observed that ZINI overexpression lines were more susceptible to infection by $P$. syringae, potentially mimicking an enhancement of ZIN1 activity by effectors.

In addition, the targeting of ZIN1 by multiple effectors indicates that ZIN1 is not just a susceptibility factor but a susceptibility hub. We recently characterized the transcriptional regulator ABSCISIC ACID REPRESSOR 1 as another susceptibility hub that interacts with HopZ1a and several other DC3000 effectors (Schreiber et al. 2020). Similar interaction hubs were identified through large-scale yeast two-hybrid screens, using effectors from $P$. syringae, the oomycete Hyaloperonspora arabidopsidis, and the fungus Golovinomyces orontii as baits against a panel of Arabidopsis proteins (Mukhtar et al. 2011; Weßling et al. 2014). These studies demonstrated that multiple effectors from the same pathogen converge on a relatively small set of host proteins and that effectors from different pathogens often target the same host proteins. Furthermore, susceptibility to $P$. syringae infection was reduced in knockouts of highly targeted proteins, such as the COP9 signalosome complex subunit 5A, the 
transcription factor TCP14, and the cell-cycle regulator APC8 (Weßling et al. 2014).

One of the challenges in defining the contribution of ZIN1 to plant immunity was the minimal previous functional annotation for this protein, so we sought to derive some inferences from analyses of the ZIN1 sequence. We scanned for conserved sequence elements and identified a TDBD near the $\mathrm{C}$ terminus of ZIN1. A small number of genes in the Arabidopsis TDBD protein family have been characterized, including ABI5binding proteins (AFPs), Increased DNA methylation 1 (IDM1), and novel interactor of JAZ (NINJA). The TDBD of NINJA interfaces with the tify domain of JAZ proteins, while an ERF-associated amphiphilic repression motif in NINJA facilitates interactions with the TOPLESS corepressor, ultimately mediating negative regulation of jasmonate-responsive gene expression (Pauwels et al. 2010). Likewise, the AFPs act as a bridge between ABI5 and either TOPLESS or histone deacetylase enzymes (Garcia et al. 2008; Lynch et al. 2017), although the absence of a tify domain in ABI5 implies that the TDBD can interact with other protein interfaces. Lastly, IDM1 is a histone acetyltransferase for which the functional relevance of the TDBD is unknown (Li et al. 2012; Qian et al. 2012). Importantly, the only conserved domain in ZIN1 is the TDBD, suggesting that ZIN1 is likely not functionally analogous to NINJA, IDM1, or the AFPs. The C-terminal portion of ZIN1, including the TDBD, is remarkably conserved in orthologous sequences throughout the plant kingdom, which implies that this region does contribute to ZIN1 function.

Our sequence analysis also revealed that most of the ZIN1 protein is predicted to be structurally disordered. Disordered regions are commonly found in DNA-binding proteins associated with transcription, DNA replication, and chromatin structure (Liu et al. 2006; Minezaki et al. 2006; Parker et al. 2019; Watson and Stott 2019). These regions influence the specificity and affinity of DNA binding (De et al. 2014; Krois et al. 2018; Vuzman and Levy 2012), mediate interactions with other proteins (Maiti et al. 2019), and serve as platforms for regulatory posttranslational modifications, such as acetylation, phosphorylation, ubiquitination, and sumoylation (Bah and Forman-Kay 2016; Krois et al. 2018). Predicted regions of disorder often contain segments that can adopt a more defined structure upon interaction with other proteins. These interaction interfaces can be SLiMs or longer stretches (MoRFs) (Cumberworth et al. 2013; Vacic et al. 2007; Weatheritt and Gibson 2012). Computational analyses of the ZIN1 sequence predicted multiple SLiMs and MoRFs, which could provide interfaces for interactions with several different effectors.

Our analyses also included structural homology modeling, which highlighted a short region at the $\mathrm{C}$ terminus of ZIN1 that resembled topoisomerase enzymes. These enzymes relieve torsional stress in DNA molecules through a process of cleavage and religation; type I topoisomerases introduce single strand breaks in DNA to facilitate relaxation of supercoiling, while type II topoisomerases accomplish this task through double-stranded DNA cleavage (Baker et al. 2009). Structural homology modeling indicated that ZIN1 was most similar to the type IB family of topoisomerases, which is compatible with our observations of ATP-independent enzymatic activity. The catalytic pentad that is typically found in these enzymes (nonsequential $\mathrm{R}, \mathrm{K}, \mathrm{R}, \mathrm{N}$, and $\mathrm{Y}$ residues) was incompletely conserved in the primary amino acid sequence of ZIN1 (Supplementary Fig. S6), although the tertiary structure of this protein could certainly include the appropriately located catalytic residues. Indeed, the region containing this pentad contributed significantly to the activity of ZIN1 in a plasmid relaxation assay (Fig. 4). Intriguingly, low levels of activity were retained in the absence of this region ( $\left.\mathrm{ZIN} 1_{1-305}\right)$, suggesting that ZIN1 may be functionally different from wellcharacterized topoisomerases. This could be influenced by the extensive structural disorder predicted for ZIN1, whereas relatively short, disordered regions are found in topoisomerase enzymes, where they are thought to help stabilize interactions with DNA (Patel et al. 2006; Vos et al. 2014). Beyond direct impacts on DNA topology, Arabidopsis topoisomerase $1 \alpha$ and some of the topoisomerase II-like microrchidia (MORC) proteins mediate RNA-directed DNA methylation to facilitate epigenetic regulation of genes and transposable elements (Brabbs et al. 2013; Dinh et al. 2014; Liu et al. 2016; Manohar et al. 2017). Altered expression of MORC proteins can significantly impact plant immunity, potentially by influencing epigenetic regulation and chromatin remodeling as well as through interactions with proteins that regulate plant immune responses (Koch et al. 2017). Similar mechanisms may be employed by ZIN1, and the extent to which these rely on topoisomerase-like activity will be the subject of future study.

For additional functional characterization of ZIN1, we examined the transcriptional profiles of WT and zinl mutant plants at two timepoints following inoculation with DC3000. In the absence of infection, significantly more genes were differentially expressed in zinl versus WT plants at 12 hpi than at 6 hpi. The differences at these two timepoints may reflect patterns of circadian regulation or they may indicate differential responses to the physical stimulus of buffer infiltration that become more pronounced over time (Baum et al. 2019; Kohler et al. 2002). Nonetheless, one gene (At1g64040) was consistently expressed at a lower level in $\mathrm{MgCl}_{2}$-infiltrated zin1 plants at both 6 and $12 \mathrm{hpi}$. This gene encodes type one protein phosphatase 3 (TOPP3), a catalytic subunit of the protein phosphatase 1 (PP1) holoenzyme. In eukaryotes, PP1 interacts with a wide range of substrates and is hypothesized to catalyze the majority of dephosphorylation events in eukaryotic cells (Bollen et al. 2010; Rebelo et al. 2015). There are nine TOPP family members in Arabidopsis, some of which directly regulate plant immunity through interactions with mitogen-activated protein kinases, although TOPP3 does not participate in these interactions (Liu et al. 2020). The impact of TOPP3 downregulation is potentially more general, however, since PP1 influences pre-mRNA processing by the spliceosome in mammalian cells (Shi et al. 2006; Ma et al. 2010), which can, in turn, affect the rate of gene transcript elongation (Herzel et al. 2017; Kornblihtt et al. 2004).

Upon exposure to DC3000, the transcriptional responses of zinl 1 and WT plants were differentiated by genes representing a broad range of biological processes. The most significant results were obtained by quantitatively comparing between host genotypes to identify APETRIZ genes. Specifically, we found that the list of effector-dependent APETRIZ " $>0$ " genes at 12 hpi was particularly enriched for GO terms associated with the ribosome and translation, most commonly encoding ribosomal subunit proteins (Table 1; Supplementary Table S14). Pathogen-responsive expression of ribosomal protein genes has been observed in other pathosystems as well. Many ribosomal protein genes are upregulated during the early stages of infection of the orchid Vanilla planifolia by Fusarium oxysporum f. sp. vanilla, perhaps to bolster the initial defense response (Solano-De la Cruz et al. 2019). The barley Mla6-mediated resistance response involves the upregulation of multiple ribosome genes (Moeller et al. 2012). Finally, tobacco cell suspensions exposed to agrobacterium initially have low levels of ribosomal protein gene expression that increase by $36 \mathrm{hpi}$ (Veena et al. 2003). Gene-silencing experiments have highlighted the significant contributions of individual ribosomal protein genes to nonhost resistance and to the execution of an ETI-associated programmed cell death response known as the 
hypersensitive response (Gabriëls et al. 2006; Lu et al. 2003; Nagaraj et al. 2016). More generally, sequencing of ribosomal protein-associated transcripts following the activation of PTI or ETI has revealed that these immune responses are tightly regulated at the translational level and are marked by significant shifts in translational efficiency for many transcripts (Meteignier et al. 2017; Xu et al. 2017; Yoo et al. 2020). It is possible that higher levels of ribosomal protein gene expression enable zinl mutant plants to better meet the translational demands for immunity-associated proteins. The increased susceptibility of ZIN1 overexpression lines may be linked to a reduced capacity for such translational reprogramming. Similarly, chloroplastrelated GO terms were highly enriched in the list of APETRIZ " $>0$ " genes at $12 \mathrm{hpi}$ (Table 1). Chloroplasts also contribute to the metabolic shifts associated with plant immunity, providing energy from photosynthesis as well as important secondary metabolites such as salicylic acid, jasmonic acid, and reactive oxygen species (Kretschmer et al. 2020; Serrano et al. 2016). Altogether, this suggests that the role of ZIN1 in mediating responses to pathogens could extend beyond the transcriptome.

Overall, we have demonstrated that ZIN1 is a susceptibility factor that interacts with several $P$. syringae effectors and that the loss of ZIN1 in Arabidopsis confers reduced susceptibility to bacterial infection. Susceptibility factors are acknowledged as an important avenue for developing disease-resistant field crops with potentially durable phenotypes (Engelhardt et al. 2018; Zaidi et al. 2018). The modification of certain susceptibility factors can have negative pleiotropic consequences, such as reduced plant fitness, but the apparent absence of such phenotypes in zinl plants suggests that this gene warrants further examination in an agriculturally relevant pathosystem under field conditions.

\section{MATERIALS AND METHODS}

\section{Cloning.}

In general, PCR amplification of sequences for cloning was performed using Phusion high-fidelity polymerase (New England BioLabs, Inc.). All oligonucleotides were supplied by Integrated DNA Technologies, Inc. and are listed in Supplementary Table S16. Following electrophoresis, PCR amplicons were purified using EZ-10 spin column gel extraction kits (Bio Basic Inc.). All restriction enzymes, as well as T4 DNA ligase for ligations, were supplied by New England BioLabs, Inc. All constructs were verified by sequencing.

Constructs for yeast two-hybrid analysis were prepared in the DupLEX-A system (OriGene Technologies, Inc.), using the vectors pEG202 and pJG4-5 to express bait and prey proteins, respectively. A collection of 23 DC3000 effectors in pEG202 was obtained as a generous gift from D. Desveaux (University of Toronto, Canada). An additional eight effectors from DC3000 were cloned into the EcoRI and XhoI sites of pEG202, and hopDl was introduced into this vector at the XhoI site alone. The EcoRI and XhoI sites of pJG4-5 were also used for cloning ZIN1 and all truncations.

For BiFC experiments, genes were cloned into the XhoI and StuI sites of previously described pBD-derived vectors (Lewis et al. 2014). These vectors provide dexamethasone-inducible expression of proteins with $\mathrm{C}$-terminal fusions to a $1 \times \mathrm{HA}$ (hemagglutinin) tag, plus either full-length YFP, nYFP (Nterminal 155 amino acids), or cYFP (C-terminal 87 amino acids). All constructs were introduced into Agrobacterium tumefaciens GV2260.

To enable ZIN1 protein purification, full-length ZIN1 and $\mathrm{ZIN}_{1-305}$ were cloned into the NcoI and BamHI sites of the expression vector $\mathrm{p} 11$, with primers designed to include a C-terminal 6xHis tag in frame with ZIN1. As a control, we cloned GUS into p11 at the NdeI and BamHI sites.

A construct for constitutive ZIN1 overexpression in Arabidopsis was assembled by cloning ZIN1-3xFlag into pENTR/DTOPO followed by Gateway cloning into pEarleyGate 100 (Earley et al. 2006).

\section{Yeast assays.}

All constructs for yeast two-hybrid analysis were transformed into yeast using Frozen-EZ yeast transformation II reagents (Zymo Research Corp.) to introduce pEG202 constructs into yeast strain RFY206 (mating type A), while pJG4-5 constructs were introduced into strain EGY48 (mating type $\alpha$ ). Transformants were recovered on synthetic defined (SD)+glucose media lacking histidine and uracil, to select for colonies containing pEG202 constructs, or lacking tryptophan, to select for pJG4-5 transformants. Yeast matings were performed in yeast extract peptone dextrose media at $30^{\circ} \mathrm{C}$ overnight, followed by two rounds of diploid selection on SD+glucoseHisUraTrp plates at $30^{\circ} \mathrm{C}$ overnight. To assay for proteinprotein interactions, diploid yeast was first resuspended in $150 \mu \mathrm{l}$ of sterile distilled water, adjusted to an optical density at $600 \mathrm{~nm}\left(\mathrm{OD}_{600}\right)$ of 2, followed by three serial 1:5 dilutions in water. Five microliters of each dilution were spotted on SD+glucose-HisUraTrpLeu and SD+galactose+ raffinose-HisUraTrpLeu plates, with yeast growth indicating the activation of a LEU2 reporter. Images of yeast plates were generally captured after 3 days of growth at $30^{\circ} \mathrm{C}$.

\section{Western blotting.}

Protein expression in yeast was evaluated following shaking incubation of cultures at $30^{\circ} \mathrm{C}$ overnight in SD+glucose-HisUra (pEG202 constructs) or SD+galactose+raffinose_Trp (pJG4-5) media. Cultures were processed for protein extraction according to a lithium acetate/sodium hydroxide pretreatment protocol described by (Zhang et al. 2011). Samples were resolved on $10 \%$ discontinuous sodium dodecyl sulfate (SDS)-polyacrylamide gel electrophoresis gels, were transferred to nitrocellulose membranes, and were detected by $\alpha$-HA (Roche), $\alpha$-Flag (SigmaAldrich), or $\alpha$-LexA antibodies (Santa Cruz Biotechnology) in combination with horseradish peroxidase-conjugated goat antirat (Santa Cruz Biotechnology) or goat antimouse (Santa Cruz Biotechnology) secondary antibodies.

\section{Plant materials and bacterial inoculations.}

Arabidopsis zinl (SALK_137325C) and zarl (SALK_013297C) T-DNA knockout lines were obtained from the Arabidopsis Biological Resource Center. The two lines were crossed to generate the zarlzinl double mutant. For plant growth, surface-sterilized Arabidopsis seeds were placed on moist Sunshine \#1 soil lacking RSi (Hassan et al. 2018) (Sun Gro Horticulture) amended with 20: 20:20 fertilizer, were stratified for 3 days at $4^{\circ} \mathrm{C}$, and were then moved to a growth room with a 9 -h photoperiod $\left(130 \mu \mathrm{E} \cdot \mathrm{m}^{-2} \mathrm{~s}^{-1}\right)$ at constant $22^{\circ} \mathrm{C}$ and $60 \%$ relative humidity. Surface-sterilized $N$. benthamiana seeds were placed on moist Sunshine Mix \#1 soil (Sun Gro) amended with 14:14:14 fertilizer and were grown under the same conditions without prior stratification.

Bacterial growth assays in Arabidopsis were performed using $P$. syringae pv. tomato DC3000, P. cannabina pv. alisalensis ES4326 (formerly $P$. syringae pv. maculicola ES4326), and $P$. syringae pv. cilantro 0788-9. Bacteria were grown overnight at $28^{\circ} \mathrm{C}$ on King's B agar media (King et al. 1954) supplemented with $50 \mu \mathrm{g}$ rifampicin per milliliter (DC3000 and 0788-9) or $300 \mu \mathrm{g}$ of streptomycin per milliliter (ES4326). For inoculations, bacteria were resuspended in $10 \mathrm{mM} \mathrm{MgCl}_{2}$, adjusted to $\mathrm{OD}_{600}=$ $0.0002\left(1 \times 10^{5} \mathrm{CFU} / \mathrm{ml}\right)$, and the suspensions were infiltrated 
into the leaves of 6-week-old Arabidopsis plants using a needleless 1-ml syringe. Quantification of in-planta bacterial growth was carried out as previously described (Katagiri et al. 2002).

For global transcriptome analysis, the leaves of 6-week-old WT or zinl Arabidopsis plants were infiltrated with $10 \mathrm{mM}$ $\mathrm{MgCl}_{2}$ or either WT DC3000 or DC3000 $h \mathrm{hrcC}$ adjusted to $\mathrm{OD}_{600}=0.2\left(1 \times 10^{8} \mathrm{CFU} / \mathrm{ml}\right)$. Tissues were harvested at 6 and $12 \mathrm{~h}$ postinfiltration for RNA extraction.

To prepare inocula for BiFC assays, agrobacterium strains were grown overnight at $28^{\circ} \mathrm{C}$ on Luria-Bertani (LB) agar media supplemented with $100 \mu \mathrm{g}$ rifampicin per milliliter and $50 \mu \mathrm{g}$ kanamycin per milliliter. Bacteria were resuspended in $1 \mathrm{ml}$ of induction media $[10 \mathrm{mM} \mathrm{MgCl} 2,10 \mathrm{mM} \mathrm{2-(N-}$ morpholino) ethanesulfonic acid (MES), $\mathrm{pH} 5.6$, and $150 \mu \mathrm{M}$ acetosyringone], were diluted with induction media so that each strain was at $\mathrm{OD}_{600}=0.45$, and were incubated at room temperature for $3 \mathrm{~h}$. For all inoculations, leaves of 5- to 7-week-old $N$. benthamiana plants were pierced with a 22-gauge needle prior to infiltration with a 1-ml needleless syringe. Protein expression was induced by spraying plants with $20 \mu \mathrm{M}$ dexamethasone at $24 \mathrm{hpi}$ and protein interaction was generally assessed $24 \mathrm{~h}$ after dexamethasone treatment.

\section{Confocal microscopy.}

Samples were prepared for BiFC analysis by using a number 4 cork borer to collect leaf discs from infiltrated areas. Leaf discs were placed in a 5-ml syringe and were infiltrated with water, then mounted on microscope slides. Images were captured using a Leica SP8 confocal laser-scanning microscope (Leica Microsystems, Inc.) equipped with a 40x waterimmersion objective (HC PL APO CS2 40x/1.10 WATER). A 514-nm argon laser line was used for YFP excitation, and fluorescence was recorded within a specific emission window of 520 to $600 \mathrm{~nm}$. Leica Application Suite X software was used for image processing.

\section{Protein purification.}

To obtain adequate yields of soluble ZIN1 protein, we transformed the expression constructs into Escherichia coli BL21(DE3) along with plasmid pG-KJE8 (Takara Bio USA, Inc.) for inducible coexpression of five different chaperone proteins. For expression, bacterial cultures were grown overnight in a $37^{\circ} \mathrm{C}$ incubator shaker in LB broth containing $20 \mu \mathrm{g}$ of chloramphenicol per milliliter and $50 \mu \mathrm{g}$ of ampicillin per milliliter. The cells were subcultured at 1:100 in LB with the same antibiotics as well as $2 \mathrm{mg}$ of L-arabinose per milliliter and $10 \mathrm{ng}$ of tetracycline per milliliter to induce chaperone coexpression. When the cultures achieved $\mathrm{OD}_{600}=0.4$, isopropyl- $\beta$-D-thiogalactoside was added to a final concentration of $0.1 \mathrm{mM}$ and the cultures were incubated with shaking at $16^{\circ} \mathrm{C}$ for approximately $20 \mathrm{~h}$. Cells were harvested by centrifugation at 2,500 $\times \mathrm{g}$ for $10 \mathrm{~min}$ and pellets were stored at $-20^{\circ} \mathrm{C}$. Both ZIN1 and GUS
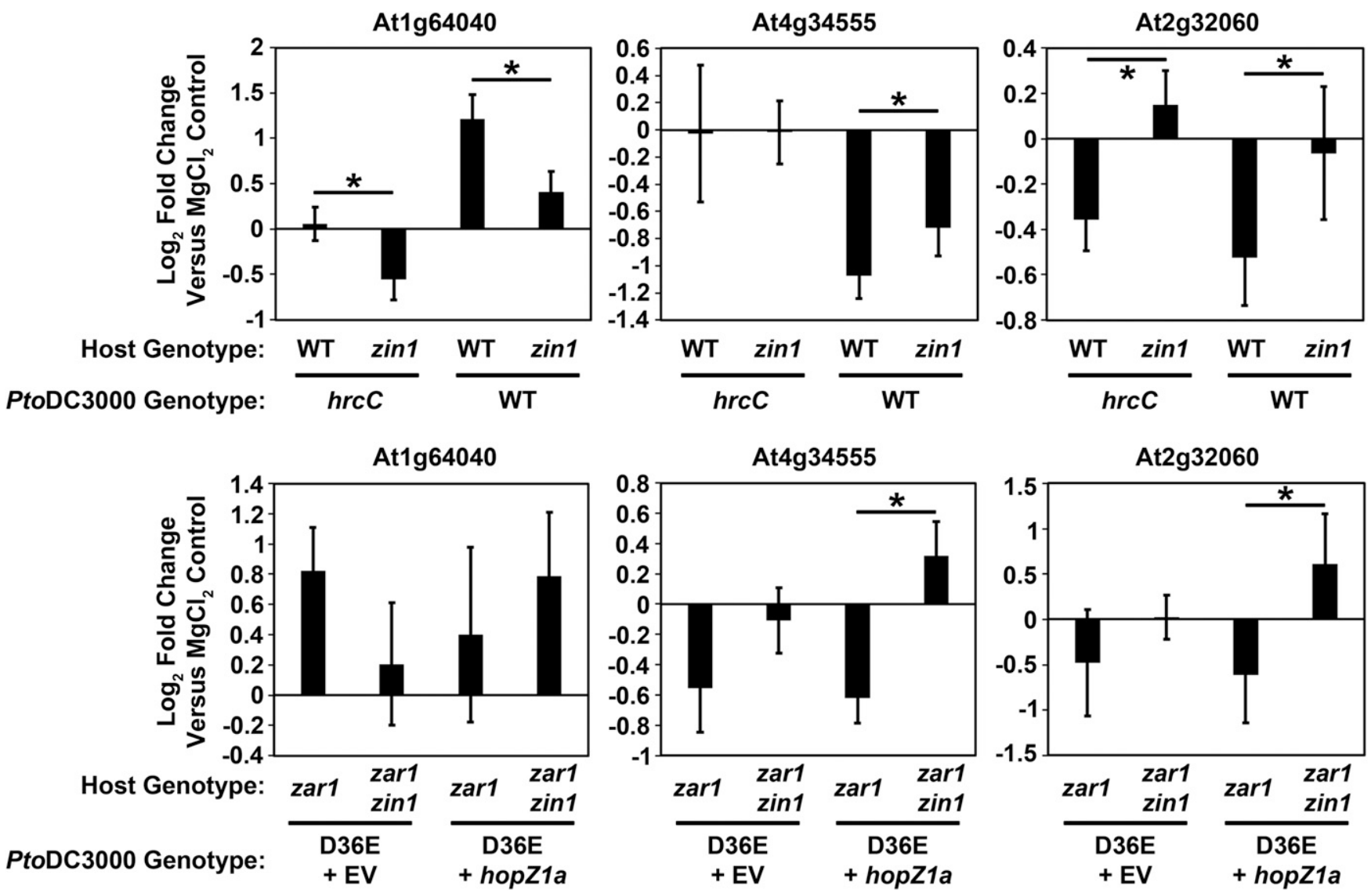

Fig. 7. HopZ1a interactor 1 (ZIN1) influences gene expression in Arabidopsis in response to inoculation with different genotypes of the pathogen Pseudomonas syringae. Six-week-old Arabidopsis plants were infiltrated with $10 \mathrm{mM} \mathrm{MgCl}{ }_{2}$ or with $1 \times 10^{8}$-CFU/ml suspensions of wild-type (WT) P. syringae pv. tomato DC3000, a type III secretion-deficient mutant $(\mathrm{hrcC})$, an effectorless $P$. syringae polymutant containing an empty expression vector (D36E+EV), or the effectorless polymutant expressing the effector HopZ1a (D36E+hopZ1a). To avoid immune recognition of HopZ1a, all D36E inoculations were performed in zarl or zarlzin1 mutant plants. Leaf tissues were collected at $12 \mathrm{~h}$ postinoculation. Following RNA extraction, real-time quantitative PCR was used to quantify the expression of specific genes normalized to a housekeeping gene (EF-1 $\alpha$ ) using the $2^{-\Delta \Delta \mathrm{Ct}}$ method. Values are presented as $\log _{2}$-fold changes relative to $\mathrm{MgCl}_{2}$-infiltrated control plants. Error bars represent standard deviation for data from at least three biological replicates and asterisks indicate a statistically significant difference between host genotypes as calculated by a Student's $t$ test $(\alpha=0.05)$. 
were affinity-purified under native conditions using Ni-NTA agarose (Qiagen), according to manufacturer instructions.

\section{Topoisomerase assays.}

Topoisomerase activity was assessed by combining plasmid DNA (4 $\mathrm{nM})$ with approximately $1 \mu \mathrm{g}$ of purified protein in a buffer containing $30 \mathrm{mM}$ Tris- $\mathrm{HCl}(\mathrm{pH} \mathrm{7.6)}, 15 \mathrm{mM} \beta$-mercaptoethanol, $8 \mathrm{mM} \mathrm{MgCl}_{2}$, and $60 \mathrm{mM} \mathrm{NaCl}$ in a total volume of $20 \mu \mathrm{l}$. Samples were incubated at $30^{\circ} \mathrm{C}$ for $1 \mathrm{~h}$. Subsequently, $1 \mu \mathrm{l}$ of $20 \%$ SDS, $0.5 \mu \mathrm{l}$ of $0.5 \mathrm{M}$ EDTA, and $1 \mu \mathrm{l}$ of proteinase $\mathrm{K}$ (Invitrogen) were added to each sample to disassociate DNAprotein complexes, and the samples were incubated at $37^{\circ} \mathrm{C}$ for $1.5 \mathrm{~h}$. Plasmid conformations were separated by electrophoresis on a $1 \%$ agarose gel at $20 \mathrm{~V}$ for 18 to $24 \mathrm{~h}$ and were visualized with GelRed nucleic acid stain (Biotium, Inc.).

\section{RNA-sequencing and expression validation.}

TRIzol reagent (Thermo Fisher Scientific, Inc.) was used to extract RNA from Arabidopsis leaves, according to manufacturer instructions. Samples were treated with DNase and were further purified using RNA clean and concentrator kits (Zymo Research Corp.) and RNA quality was assessed on an Agilent 2100 Bioanalyzer (Agilent). Library preparation and sequencing were performed by the University of California Davis Genome Center, yielding 90-bp single-end reads from an Illumina Hiseq 4000 sequencer (Illumina, Inc.). All RNA-seq data are accessible from the Gene Expression Omnibus database at the National Center for Biotechnology Information as series GSE158780.

To evaluate the expression of specific genes, cDNA was generated with Superscript III reverse transcription (Thermo Fisher Scientific) and anchored oligo-dT primers. RT-qPCR was performed on a Bio-Rad CFX96 thermocycler (Bio-Rad Laboratories, Inc.) using EvaGreen fluorescent dye (Biotium, Inc.) and ExTaq polymerase (Takara Bio USA Inc.).

\section{Data analysis.}

Most of the RNA-seq data analysis was conducted in the Galaxy environment (available online). Read quality was assessed with FastQC (Andrews 2010), and the first 12 nucleotides of each read were removed with Trimmomatic (Bolger et al. 2014). Reads were aligned to the Arabidopsis TAIR10 genome with either STAR (Dobin et al. 2013) or HISAT2 (Kim et al. 2015) and read counts were determined by the program featureCounts (Liao et al. 2014). From these counts, differential gene expression was analyzed by either the edgeR (Robinson et al. 2010) or limma-voom algorithm (Law et al. 2014). For both of these analyses, genes with less than one count per million in any sample were filtered out, and the threshold for differential expression was set at a $\log _{2}$-fold change of 1 with a false discovery rate (for edgeR) or adjusted $P$ value (for limma-voom) of less than 0.05. Lists of differentially expressed genes were parsed and were further analyzed in R (R Core Team 2019). The significance of overlap between datasets of differentially expressed genes was calculated as an exact hypergeometric probability (Kim et al. 2001) using an online Nemates resource.

A variety of resources were utilized to analyze various features of the ZIN1 amino acid sequence. Conserved domains were identified by Pfam (El-Gebali et al. 2019), and structural homology modeling was performed using Phyre2 (Kelley et al. 2015). Secondary structure was predicted by PSIPRED (Buchan and Jones 2019). A consensus of results from PONDR-FITVSL2 (Xue et al. 2010) and DISOPRED3 (Jones and Cozzetto 2015) defined regions of probable structural disorder. Putative interaction interfaces were predicted using the Eukaryotic Linear Motif resource (Gouw et al. 2018), ANCHOR2 (Mészáros et al. 2018), MoRFpred (Disfani et al. 2012), and MoRF chibi (Malhis and Gsponer 2015; Malhis et al. 2016). We prepared an alignment of ZIN1 and a Deinococcus radiodurans topoisomerase using ClustalW (Thompson et al. 1994) in MEGA-X (Kumar et al. 2018) and formatted the alignment using BoxShade. A phylogenetic tree of TDBD proteins was also generated in MEGA-X, using a maximum likelihood method.

\section{Accession numbers.}

Sequence data for the genes examined in this study can be retrieved from the National Center for Biotechnology Information using accession numbers NM_125375 (ZIN1) and NM_114527 (GRAS1).

\section{ACKNOWLEDGMENTS}

We thank R. Rubalcava and D. Ouyang, for technical assistance with the initial characterization of candidate HopZ1a interactors, as well as $\mathrm{J}$. Hassan for critically reading the manuscript. We thank D. Desveaux for sharing plasmids with us, F. Harmon for advice on transcriptome analyses, and the Arabidopsis Biological Resource Center for seeds.

\section{AUTHOR-RECOMMENDED INTERNET RESOURCES}

BoxShade: https://embnet.vital-it.ch/software/BOX_form.html Galaxy tools: https://usegalaxy.org

Nemates resource: http://nemates.org/MA/progs/overlap_stats.html

\section{LITERATURE CITED}

Andrews, S. 2010. FastQC: A quality control tool for high throughput sequence data. Babraham Bioinformatics, Cambridge. Published online. https://www.bioinformatics.babraham.ac.uk/projects/fastqc

Bah, A., and Forman-Kay, J. D. 2016. Modulation of intrinsically disordered protein function by post-translational modifications. J. Biol. Chem. 291:6696-6705.

Bai, Y., Meng, Y., Huang, D., Qi, Y., and Chen, M. 2011. Origin and evolutionary analysis of the plant-specific TIFY transcription factor family. Genomics 98:128-136.

Baker, N. M., Rajan, R., and Mondragón, A. 2009. Structural studies of type I topoisomerases. Nucleic Acids Res. 37:693-701.

Baum, S., Reimer-Michalski, E. M., Bolger, A., Mantai, A. J., Benes, V., Usadel, B., and Conrath, U. 2019. Isolation of open chromatin identifies regulators of systemic acquired resistance. Plant Physiol. 181:817-833.

Bernsdorff, F., Döring, A. C., Gruner, K., Schuck, S., Bräutigam, A., and Zeier, J. 2016. Pipecolic acid orchestrates plant systemic acquired resistance and defense priming via salicylic acid-dependent and -independent pathways. Plant Cell 28:102-129.

Bolger, A. M., Lohse, M., and Usadel, B. 2014. Trimmomatic: A flexible trimmer for Illumina sequence data. Bioinformatics 30:2114-2120.

Bollen, M., Peti, W., Ragusa, M. J., and Beullens, M. 2010. The extended PP1 toolkit: Designed to create specificity. Trends Biochem. Sci. 35:450-458.

Bottomley, M. J., Collard, M. W., Huggenvik, J. I., Liu, Z., Gibson, T. J., and Sattler, M. 2001. The SAND domain structure defines a novel DNAbinding fold in transcriptional regulation. Nat. Struct. Biol. 8:626-633.

Brabbs, T. R., He, Z., Hogg, K., Kamenski, A., Li, Y., Paszkiewicz, K. H., Moore, K. A., O'Toole, P., Graham, I. A., and Jones, L. 2013. The stochastic silencing phenotype of Arabidopsis morc6 mutants reveals a role in efficient RNA-directed DNA methylation. Plant J. 75:836-846.

Buchan, D. W. A., and Jones, D. T. 2019. The PSIPRED protein analysis workbench: 20 years on. Nucleic Acids Res. 47 (W1):W402-W407.

Carles, C. C., Choffnes-Inada, D., Reville, K., Lertpiriyapong, K., and Fletcher, J. C. 2005. ULTRAPETALA1 encodes a SAND domain putative transcriptional regulator that controls shoot and floral meristem activity in Arabidopsis. Development 132:897-911.

Cumberworth, A., Lamour, G., Babu, M. M., and Gsponer, J. 2013. Promiscuity as a functional trait: Intrinsically disordered regions as central players of interactomes. Biochem. J. 454:361-369.

De, S., Chan, A. C. K., Coyne, H. J., 3rd, Bhachech, N., Hermsdorf, U., Okon, M., Murphy, M. E. P., Graves, B. J., and McIntosh, L. P. 2014 Steric mechanism of auto-inhibitory regulation of specific and nonspecific DNA binding by the ETS transcriptional repressor ETV6. J. Mol. Biol. 426:1390-1406.

Ding, B., and Wang, G.-L. 2015. Chromatin versus pathogens: The function of epigenetics in plant immunity. Front. Plant Sci. 6:675.

Dinh, T. T., Gao, L., Liu, X., Li, D., Li, S., Zhao, Y., O’Leary, M., Le, B., Schmitz, R. J., Manavella, P. A., Li, S., Weigel, D., Pontes, O., Ecker, J. R., 
and Chen, X. 2014. DNA topoisomerase $1 \alpha$ promotes transcriptional silencing of transposable elements through DNA methylation and histone lysine 9 dimethylation in Arabidopsis. PLoS Genet. 10:e1004446.

Disfani, F. M., Hsu, W. L., Mizianty, M. J., Oldfield, C. J., Xue, B., Dunker, A. K., Uversky, V. N., and Kurgan, L. 2012. MoRFpred, a computational tool for sequence-based prediction and characterization of short disorder-to-order transitioning binding regions in proteins. Bioinformatics 28:i75-i83.

Dobin, A., Davis, C. A., Schlesinger, F., Drenkow, J., Zaleski, C., Jha, S., Batut, P., Chaisson, M., and Gingeras, T. R. 2013. STAR: Ultrafast universal RNA-seq aligner. Bioinformatics 29:15-21.

Earley, K. W., Haag, J. R., Pontes, O., Opper, K., Juehne, T., Song, K., and Pikaard, C. S. 2006. Gateway-compatible vectors for plant functional genomics and proteomics. Plant J. 45:616-629.

El-Gebali, S., Mistry, J., Bateman, A., Eddy, S. R., Luciani, A., Potter, S. C. Qureshi, M., Richardson, L. J., Salazar, G. A., Smart, A., Sonnhammer, E. L. L., Hirsh, L., Paladin, L., Piovesan, D., Tosatto, S. C. E., and Finn, R. D. 2019. The Pfam protein families database in 2019. Nucleic Acids Res. 47 (D1):D427-D432.

Engelhardt, S., Stam, R., and Hückelhoven, R. 2018. Good riddance? Breaking disease susceptibility in the era of new breeding technologies. Agronomy (Basel) 8:114.

Gabriëls, S. H. E. J., Takken, F. L. W., Vossen, J. H., de Jong, C. F., Liu, Q., Turk, S. C. H. J., Wachowski, L. K., Peters, J., Witsenboer, H. M. A., de Wit, P. J. G. M., and Joosten, M. H. A. J. 2006. cDNA-AFLP combined with functional analysis reveals novel genes involved in the hypersensitive response. Mol. Plant-Microbe Interact. 19:567-576.

Garcia, M. E., Lynch, T., Peeters, J., Snowden, C., and Finkelstein, R. 2008 A small plant-specific protein family of ABI five binding proteins (AFPs) regulates stress response in germinating Arabidopsis seeds and seedlings. Plant Mol. Biol. 67:643-658.

Gouw, M., Michael, S., Sámano-Sánchez, H., Kumar, M., Zeke, A., Lang, B., Bely, B., Chemes, L. B., Davey, N. E., Deng, Z., Diella, F., Gürth, C. M., Huber, A. K., Kleinsorg, S., Schlegel, L. S., Palopoli, N., Roey, K. V., Altenberg, B., Reményi, A., Dinkel, H., and Gibson, T. J. 2018 The eukaryotic linear motif resource-2018 update. Nucleic Acids Res. 46 (D1):D428-D434

Hassan, J. A., de la Torre-Roche, R., White, J. C., and Lewis, J. D. 2018 Soil mixture composition alters Arabidopsis susceptibility to Pseudomonas syringae infection. Plant Direct 2:e0044.

Herzel, L., Ottoz, D. S. M., Alpert, T., and Neugebauer, K. M. 2017 Splicing and transcription touch base: Co-transcriptional spliceosome assembly and function. Nat. Rev. Mol. Cell Biol. 18:637-650.

Huang, W., Sherman, B. T., and Lempicki, R. A. 2009a. Bioinformatics enrichment tools: Paths toward the comprehensive functional analysis of large gene lists. Nucleic Acids Res. 37:1-13

Huang, W., Sherman, B. T., and Lempicki, R. A. 2009b. Systematic and integrative analysis of large gene lists using DAVID bioinformatics resources. Nat. Protoc. 4:44-57.

Jones, D. T., and Cozzetto, D. 2015. DISOPRED3: Precise disordered region predictions with annotated protein-binding activity. Bioinformatics 31:857-863

Katagiri, F., Thilmony, R., and He, S. Y. 2002. The Arabidopsis thalianaPseudomonas syringae interaction. Pages 1-35 in: The Arabidopsis Book. Somerville, C. R., and Meyerowitz, E. M., eds. American Society of Plant Biologists, Rockville.

Kazan, K., and Manners, J. M. 2012. JAZ repressors and the orchestration of phytohormone crosstalk. Trends Plant Sci. 17:22-31.

Kelley, L. A., Mezulis, S., Yates, C. M., Wass, M. N., and Sternberg, M. J. E. 2015. The Phyre2 web portal for protein modeling, prediction and analysis. Nat. Protoc. 10:845-858.

Khan, M., Seto, D., Subramaniam, R., and Desveaux, D. 2018. Oh, the places they'll go! A survey of phytopathogen effectors and their host targets. Plant J. 93:651-663.

Kim, D., Langmead, B., and Salzberg, S. L. 2015. HISAT: A fast spliced aligner with low memory requirements. Nat. Methods 12:357-360.

Kim, J.-G., Stork, W., and Mudgett, M. B. 2013. Xanthomonas type III effector XopD desumoylates tomato transcription factor SIERF4 to suppress ethylene responses and promote pathogen growth. Cell Host Microbe 13:143-154.

Kim, S. K., Lund, J., Kiraly, M., Duke, K., Jiang, M., Stuart, J. M., Eizinger, A., Wylie, B. N., and Davidson, G. S. 2001. A gene expression map for Caenorhabditis elegans. Science 293:2087-2092.

King, E. O., Ward, M. K., and Raney, D. E. 1954. Two simple media for the demonstration of pyocyanin and fluorescin. J. Lab. Clin. Med. 44:301-307.

Klessig, D. F., Choi, H. W., and Dempsey, D. A. 2018. Systemic acquired resistance and salicylic acid: Past, present, and future. Mol. PlantMicrobe Interact. 31:871-888.
Koch, A., Kang, H. G., Steinbrenner, J., Dempsey, D. A., Klessig, D. F., and Kogel, K. H. 2017. MORC proteins: Novel players in plant and animal health. Front. Plant Sci. 8:1720.

Kohler, A., Schwindling, S., and Conrath, U. 2002. Benzothiadiazoleinduced priming for potentiated responses to pathogen infection, wounding, and infiltration of water into leaves requires the NPRI/NIMI gene in Arabidopsis. Plant Physiol. 128:1046-1056.

Kong, L., Qiu, X., Kang, J., Wang, Y., Chen, H., Huang, J., Qiu, M., Zhao, Y., Kong, G., Ma, Z., Wang, Y., Ye, W., Dong, S., Ma, W., and Wang, Y. 2017. A Phytophthora effector manipulates host histone acetylation and reprograms defense gene expression to promote infection. Curr. Biol. 27: 981-991.

Kornblihtt, A. R., de la Mata, M., Fededa, J. P., Muñoz, M. J., and Nogués, G. 2004. Multiple links between transcription and splicing. RNA 10 1489-1498.

Kretschmer, M., Damoo, D., Djamei, A., and Kronstad, J. 2020. Chloroplasts and plant immunity: Where are the fungal effectors? Pathogens 9: 19.

Krois, A. S., Dyson, H. J., and Wright, P. E. 2018. Long-range regulation of p53 DNA binding by its intrinsically disordered Nterminal transactivation domain. Proc. Natl. Acad. Sci. U.S.A. 115 E11302-E11310.

Kumar, S., Stecher, G., Li, M., Knyaz, C., and Tamura, K. 2018. MEGA X: Molecular evolutionary genetics analysis across computing platforms. Mol. Biol. Evol. 35:1547-1549.

Kusch, S., and Panstruga, R. 2017. mlo-based resistance: An apparently universal "weapon" to defeat powdery mildew disease. Mol. PlantMicrobe Interact. 30:179-189.

Law, C. W., Chen, Y., Shi, W., and Smyth, G. K. 2014. voom: Precision weights unlock linear model analysis tools for RNA-seq read counts Genome Biol. 15:R29.

Lee, A. H. Y., Hurley, B., Felsensteiner, C., Yea, C., Ckurshumova, W. Bartetzko, V., Wang, P. W., Quach, V., Lewis, J. D., Liu, Y. C., Börnke, F., Angers, S., Wilde, A., Guttman, D. S., and Desveaux, D. 2012. A bacterial acetyltransferase destroys plant microtubule networks and blocks secretion. PLoS Pathog. 8:e1002523.

Lewis, J. D., Guttman, D. S., and Desveaux, D. 2009. The targeting of plant cellular systems by injected type III effector proteins. Semin. Cell Dev. Biol. 20:1055-1063.

Lewis, J. D., Lee, A. H.-Y., Hassan, J. A., Wan, J., Hurley, B., Jhingree, J. R., Wang, P. W., Lo, T., Youn, J.-Y., Guttman, D. S., and Desveaux, D. 2013. The Arabidopsis ZED1 pseudokinase is required for ZAR1mediated immunity induced by the Pseudomonas syringae type III effector HopZ1a. Proc. Natl. Acad. Sci. U.S.A. 110:18722-18727.

Lewis, J. D., Wan, J., Ford, R., Gong, Y., Fung, P., Nahal, H., Wang, P. W., Desveaux, D., and Guttman, D. S. 2012. Quantitative interactor screening with next-generation sequencing (QIS-Seq) identifies Arabidopsis thaliana MLO2 as a target of the Pseudomonas syringae type III effector HopZ2. BMC Genomics 13:8.

Lewis, J. D., Wilton, M., Mott, G. A., Lu, W., Hassan, J. A., Guttman, D. S., and Desveaux, D. 2014. Immunomodulation by the Pseudomonas syringae HopZ type III effector family in Arabidopsis. PLoS One 9: e116152.

Lewis, J. D., Wu, R., Guttman, D. S., and Desveaux, D. 2010. Allelespecific virulence attenuation of the Pseudomonas syringae HopZ1a type III effector via the Arabidopsis ZAR1 resistance protein. PLoS Genet. 6:e1000894.

Li, H., Wang, H., Jing, M., Zhu, J., Guo, B., Wang, Y., Lin, Y., Chen, H., Kong, L., Ma, Z., Wang, Y., Ye, W., Dong, S., Tyler, B., and Wang, Y. 2018. A Phytophthora effector recruits a host cytoplasmic transacetylase into nuclear speckles to enhance plant susceptibility. eLife 7:e40039.

Li, X., Qian, W., Zhao, Y., Wang, C., Shen, J., Zhu, J.-K., and Gong, Z. 2012. Antisilencing role of the RNA-directed DNA methylation pathway and a histone acetyltransferase in Arabidopsis. Proc. Natl. Acad. Sci. U.S.A. 109:11425-11430.

Liao, Y., Smyth, G. K., and Shi, W. 2014. featureCounts: An efficient general purpose program for assigning sequence reads to genomic features. Bioinformatics 30:923-930.

Liu, J., Perumal, N. B., Oldfield, C. J., Su, E. W., Uversky, V. N., and Dunker, A. K. 2006. Intrinsic disorder in transcription factors. Biochemistry 45:6873-6888

Liu, Y., Yan, J., Qin, Q., Zhang, J., Chen, Y., Zhao, L., He, K., and Hou, S. 2020. Type one protein phosphatases (TOPPs) contribute to the plant defense response in Arabidopsis. J. Integr. Plant Biol. 62:360-377.

Liu, Z.-W., Zhou, J.-X., Huang, H.-W., Li, Y.-Q., Shao, C.-R., Li, L., Cai, T., Chen, S., and He, X.-J. 2016. Two components of the RNA-directed DNA methylation pathway associate with MORC6 and silence loci targeted by MORC6 in Arabidopsis. PLoS Genet. 12:e1006026. 
Lohman, B. K., Weber, J. N., and Bolnick, D. I. 2016. Evaluation of TagSeq, a reliable low-cost alternative for RNAseq. Mol. Ecol. Resour. 16:1315-1321.

Lolle, S., Stevens, D., and Coaker, G. 2020. Plant NLR-triggered immunity: From receptor activation to downstream signaling. Curr. Opin. Immunol. 62:99-105.

Lu, R., Malcuit, I., Moffett, P., Ruiz, M. T., Peart, J., Wu, A. J., Rathjen, J. P., Bendahmane, A., Day, L., and Baulcombe, D. C. 2003. High throughput virus-induced gene silencing implicates heat shock protein 90 in plant disease resistance. EMBO J. 22:5690-5699.

Lynch, T. J., Erickson, B. J., Miller, D. R., and Finkelstein, R. R. 2017. ABI5-binding proteins (AFPs) alter transcription of ABA-induced genes via a variety of interactions with chromatin modifiers. Plant Mol. Biol. 93:403-418.

Ma, C.-T., Ghosh, G., Fu, X.-D., and Adams, J. A. 2010. Mechanism of dephosphorylation of the SR protein ASF/SF2 by protein phosphatase 1 . J. Mol. Biol. 403:386-404.

Maiti, S., Acharya, B., Boorla, V. S., Manna, B., Ghosh, A., and De, S. 2019. Dynamic studies on intrinsically disordered regions of two paralogous transcription factors reveal rigid segments with important biological functions. J. Mol. Biol. 431:1353-1369.

Malhis, N., and Gsponer, J. 2015. Computational identification of MoRFs in protein sequences. Bioinformatics 31:1738-1744.

Malhis, N., Jacobson, M., and Gsponer, J. 2016. MoRFchibi SYSTEM: Software tools for the identification of MoRFs in protein sequences. Nucleic Acids Res. 44:W483-W493.

Manohar, M., Choi, H. W., Manosalva, P., Austin, C. A., Peters, J. E., and Klessig, D. F. 2017. Plant and human MORC proteins have DNAmodifying activities similar to type II topoisomerases, but require one or more additional factors for full activity. Mol. Plant-Microbe Interact. 30: 87-100.

McLellan, H., Boevink, P. C., Armstrong, M. R., Pritchard, L., Gomez, S., Morales, J., Whisson, S. C., Beynon, J. L., and Birch, P. R. J. 2013. An RxLR effector from Phytophthora infestans prevents re-localisation of two plant NAC transcription factors from the endoplasmic reticulum to the nucleus. PLoS Pathog. 9:e1003670.

Mészáros, B., Erdös, G., and Dosztányi, Z. 2018. IUPred2A: Contextdependent prediction of protein disorder as a function of redox state and protein binding. Nucleic Acids Res. 46 (W1):W329-W337.

Meteignier, L. V., El Oirdi, M., Cohen, M., Barff, T., Matteau, D., Lucier, J. F., Rodrigue, S., Jacques, P. E., Yoshioka, K., and Moffett, P. 2017. Translatome analysis of an NB-LRR immune response identifies important contributors to plant immunity in Arabidopsis. J. Exp. Bot. 68:2333-2344.

Meyer, E., Aglyamova, G. V., and Matz, M. V. 2011. Profiling gene expression responses of coral larvae (Acropora millepora) to elevated temperature and settlement inducers using a novel RNA-Seq procedure. Mol. Ecol. 20:3599-3616.

Minezaki, Y., Homma, K., Kinjo, A. R., and Nishikawa, K. 2006. Human transcription factors contain a high fraction of intrinsically disordered regions essential for transcriptional regulation. J. Mol. Biol. 359:1137-1149.

Moeller, J. R., Moscou, M. J., Bancroft, T., Skadsen, R. W., Wise, R. P., and Whitham, S. A. 2012. Differential accumulation of host mRNAs on polyribosomes during obligate pathogen-plant interactions. Mol. Biosyst. 8:2153-2165.

Mukhtar, M. S., Carvunis, A.-R., Dreze, M., Epple, P., Steinbrenner, J., Moore, J., Tasan, M., Galli, M., Hao, T., Nishimura, M. T., Pevzner, S. J., Donovan, S. E., Ghamsari, L., Santhanam, B., Romero, V., Poulin, M. M., Gebreab, F., Gutierrez, B. J., Tam, S., Monachello, D., Boxem, M., Harbort, C. J., McDonald, N., Gai, L., Chen, H., He, Y., Vandenhaute, J., Roth, F. P., Hill, D. E., Ecker, J. R., Vidal, M., Beynon, J., Braun, P., and Dangl, J. L. 2011. Independently evolved virulence effectors converge onto hubs in a plant immune system network. Science 333:596-601.

Nagaraj, S., Senthil-Kumar, M., Ramu, V. S., Wang, K., and Mysore, K. S. 2016. Plant ribosomal proteins, RPL12 and RPL19, play a role in nonhost disease resistance against bacterial pathogens. Front. Plant Sci. 6:1192.

Parker, M. W., Bell, M., Mir, M., Kao, J. A., Darzacq, X., Botchan, M. R., and Berger, J. M. 2019. A new class of disordered elements controls DNA replication through initiator self-assembly. eLife 8:e48562.

Patel, A., Shuman, S., and Mondragón, A. 2006. Crystal structure of a bacterial type IB DNA topoisomerase reveals a preassembled active site in the absence of DNA. J. Biol. Chem. 281:6030-6037.

Pauwels, L., Barbero, G. F., Geerinck, J., Tilleman, S., Grunewald, W., Pérez, A. C., Chico, J. M., Bossche, R. V., Sewell, J., Gil, E., GarcíaCasado, G., Witters, E., Inzé, D., Long, J. A., De Jaeger, G., Solano, R., and Goossens, A. 2010. NINJA connects the co-repressor TOPLESS to jasmonate signalling. Nature 464:788-791.
Peng, Y., van Wersch, R., and Zhang, Y. 2018. Convergent and divergent signaling in PAMP-triggered immunity and effector-triggered immunity. Mol. Plant-Microbe Interact. 31:403-409.

Qian, W., Miki, D., Zhang, H., Liu, Y., Zhang, X., Tang, K., Kan, Y., La, H., Li, X., Li, S., Zhu, X., Shi, X., Zhang, K., Pontes, O., Chen, X., Liu, R., Gong, Z., and Zhu, J.-K. 2012. A histone acetyltransferase regulates active DNA demethylation in Arabidopsis. Science 336:1445-1448.

Qin, J., Wang, K., Sun, L., Xing, H., Wang, S., Li, L., Chen, S., Guo, H. S., and Zhang, J. 2018. The plant-specific transcription factors CBP60g and SARD1 are targeted by a Verticillium secretory protein VdSCP41 to modulate immunity. eLife 7:e34902.

R Core Team. 2019. R: A language and environment for statistical computing. R Foundation for Statistical Computing, Vienna. https: //www.R-project.org/.

Rebelo, S., Santos, M., Martins, F., da Cruz e Silva, E. F., and da Cruz e Silva, O. A. 2015. Protein phosphatase 1 is a key player in nuclear events. Cell. Signal. 27:2589-2598.

Robinson, M. D., McCarthy, D. J., and Smyth, G. K. 2010. edgeR: A Bioconductor package for differential expression analysis of digital gene expression data. Bioinformatics 26:139-140.

Saijo, Y., Loo, E. P., and Yasuda, S. 2018. Pattern recognition receptors and signaling in plant-microbe interactions. Plant J. 93:592-613.

Sanchez, R., and Zhou, M.-M. 2011. The PHD finger: A versatile epigenome reader. Trends Biochem. Sci. 36:364-372.

Schreiber, K. J., Baudin, M., Hassan, J. A., and Lewis, J. D. 2016. Die another day: Molecular mechanisms of effector-triggered immunity elicited by type III secreted effector proteins. Semin. Cell Dev. Biol. 56: 124-133.

Schreiber, K. J., Hassan, J. A., and Lewis, J. D. 2020. Arabidopsis abscisic acid repressor 1 (ABR1) is a susceptibility hub that interacts with multiple Pseudomonas syringae effectors. Plant J. tpj.15110.

Serrano, I., Audran, C., and Rivas, S. 2016. Chloroplasts at work during plant innate immunity. J. Exp. Bot. 67:3845-3854.

Shan, L., Oh, H. S., Chen, J., Guo, M., Zhou, J., Alfano, J. R., Collmer, A., Jia, X., and Tang, X. 2004. The HopPtoF locus of Pseudomonas syringae pv. tomato DC3000 encodes a type III chaperone and a cognate effector. Mol. Plant-Microbe Interact. 17:447-455.

Shi, Y., Reddy, B., and Manley, J. L. 2006. PP1/PP2A phosphatases are required for the second step of Pre-mRNA splicing and target specific snRNP proteins. Mol. Cell 23:819-829.

Shine, M. B., Xiao, X., Kachroo, P., and Kachroo, A. 2019. Signaling mechanisms underlying systemic acquired resistance to microbial pathogens. Plant Sci. 279:81-86.

Solano-De la Cruz, M. T., Adame-García, J., Gregorio-Jorge, J., JiménezJacinto, V., Vega-Alvarado, L., Iglesias-Andreu, L. G., EscobarHernández, E. E., and Luna-Rodríguez, M. 2019. Functional categorization of de novo transcriptome assembly of Vanilla planifolia Jacks. potentially points to a translational regulation during early stages of infection by Fusarium oxysporum f. sp. vanillae. BMC Genomics 20: 826.

Thompson, J. D., Higgins, D. G., and Gibson, T. J. 1994. CLUSTAL W: Improving the sensitivity of progressive multiple sequence alignment through sequence weighting, position-specific gap penalties and weight matrix choice. Nucleic Acids Res. 22:4673-4680.

Turnbull, D., Wang, H., Breen, S., Malec, M., Naqvi, S., Yang, L., Welsh, L., Hemsley, P., Zhendong, T., Brunner, F., Gilroy, E. M., and Birch, P. R. J. 2019. AVR2 targets BSL family members, which act as susceptibility factors to suppress host immunity. Plant Physiol. 180: 571-581.

Turnbull, D., Yang, L., Naqvi, S., Breen, S., Welsh, L., Stephens, J., Morris, J., Boevink, P. C., Hedley, P. E., Zhan, J., Birch, P. R. J., and Gilroy, E. M. 2017. RXLR effector AVR2 up-regulates a brassinosteroid-responsive bHLH transcription factor to suppress immunity. Plant Physiol. 174: 356-369.

Vacic, V., Oldfield, C. J., Mohan, A., Radivojac, P., Cortese, M. S., Uversky, V. N., and Dunker, A. K. 2007. Characterization of molecular recognition features, MoRFs, and their binding partners. J. Proteome Res. 6:2351-2366.

van Schie, C. C. N., and Takken, F. L. W. 2014. Susceptibility genes 101: How to be a good host. Annu. Rev. Phytopathol. 52:551-581.

Varden, F. A., De la Concepcion, J. C., Maidment, J. H., and Banfield, M. J. 2017. Taking the stage: Effectors in the spotlight. Curr. Opin. Plant Biol. 38:25-33.

Veena, Jiang, H., Doerge, R. W., and Gelvin, S. B. 2003. Transfer of T-DNA and Vir proteins to plant cells by Agrobacterium tumefaciens induces expression of host genes involved in mediating transformation and suppresses host defense gene expression. Plant J. 35: 219-236. 
Verdier, V., Triplett, L. R., Hummel, A. W., Corral, R., Cernadas, R. A., Schmidt, C. L., Bogdanove, A. J., and Leach, J. E. 2012. Transcription activator-like (TAL) effectors targeting OsSWEET genes enhance virulence on diverse rice (Oryza sativa) varieties when expressed individually in a TAL effector-deficient strain of Xanthomonas oryzae. New Phytol. 196:1197-1207.

Vos, S. M., Lyubimov, A. Y., Hershey, D. M., Schoeffler, A. J., Sengupta, S., Nagaraja, V., and Berger, J. M. 2014. Direct control of type IIA topoisomerase activity by a chromosomally encoded regulatory protein. Genes Dev. 28:1485-1497.

Vuzman, D., and Levy, Y. 2012. Intrinsically disordered regions as affinity tuners in protein-DNA interactions. Mol. Biosyst. 8:47-57.

Watson, M., and Stott, K. 2019. Disordered domains in chromatin-binding proteins. Essays Biochem. 63:147-156.

Weatheritt, R. J., and Gibson, T. J. 2012. Linear motifs: Lost in (pre) translation. Trends Biochem. Sci. 37:333-341.

Wei, H.-L., Chakravarthy, S., Mathieu, J., Helmann, T. C., Stodghill, P., Swingle, B., Martin, G. B., and Collmer, A. 2015. Pseudomonas syringae pv. tomato DC3000 type III secretion effector polymutants reveal an interplay between HopAD1 and AvrPtoB. Cell Host Microbe 17:752-762.

Weßling, R., Epple, P., Altmann, S., He, Y., Yang, L., Henz, S. R., McDonald, N., Wiley, K., Bader, K. C., Gläßer, C., Mukhtar, M. S., Haigis, S., Ghamsari, L., Stephens, A. E., Ecker, J. R., Vidal, M., Jones,
J. D. G., Mayer, K. F. X., Ver Loren van Themaat, E., Weigel, D., Schulze-Lefert, P., Dangl, J. L., Panstruga, R., and Braun, P. 2014. Convergent targeting of a common host protein-network by pathogen effectors from three kingdoms of life. Cell Host Microbe 16:364-375.

Xu, G., Greene, G. H., Yoo, H., Liu, L., Marqués, J., Motley, J., and Dong, X. 2017. Global translational reprogramming is a fundamental layer of immune regulation in plants. Nature 545:487-490.

Xue, B., Dunbrack, R. L., Williams, R. W., Dunker, A. K., and Uversky, V. N. 2010. PONDR-FIT: A meta-predictor of intrinsically disordered amino acids. Biochim. Biophys. Acta 1804:996-1010.

Yang, L., Teixeira, P. J. P. L., Biswas, S., Finkel, O. M., He, Y., SalasGonzalez, I., English, M. E., Epple, P., Mieczkowski, P., and Dangl, J. L. 2017. Pseudomonas syringae type III effector HopBB1 promotes host transcriptional repressor degradation to regulate phytohormone responses and virulence. Cell Host Microbe 21:156-168.

Yoo, H., Greene, G. H., Yuan, M., Xu, G., Burton, D., Liu, L., Marqués, J., and Dong, X. 2020. Translational regulation of metabolic dynamics during effector-triggered immunity. Mol. Plant 13:88-98.

Zaidi, S. S. A., Mukhtar, M. S., and Mansoor, S. 2018. Genome editing: Targeting susceptibility genes for plant disease resistance. Trends Biotechnol. 36:898-906.

Zhang, T., Lei, J., Yang, H., Xu, K., Wang, R., and Zhang, Z. 2011. An improved method for whole protein extraction from yeast Saccharomyces cerevisiae. Yeast 28:795-798. 